\title{
A Computer Program POWNOR for Fitting the Power-Normal and -Lognormal Models to Life or Strength Data from Specimens of Various Sizes
}

\author{
Wayne Nelson \\ NIST/NSF/ASA Senior Research Fellow \\ and private Consultant \\ Schenectady, NY

\section{Necip Dognanksoy} \\ NIST/NSF/ASA Associate Research Fellow \\ and General Electric Co. \\ Schenectady, NY
}

U.S. DEPARTMENT OF COMMERCE Technology Administration Nationa! Institute of Standards and Technology

Center for Computing and Applied Mathematics Statistical Engineering Division Gaithersburg, MD 20899

U.S. DEPARTMENT OF COMMERCE Barbara Hackman Franklin, Secretary NATIONAL INSTITUTE OF STANDARDS AND TECHNOLOGY John W. Lyons, Director 



\section{A Computer Program POWNOR for Fitting the Power-Normal and -Lognormal Models to Life or Strength Data from Specimens of Various Sizes}

\section{Wayne Nelson}

NIST/NSF/ASA Senior Research Fellow and Private Consultant Schenectady, NY

\section{Necip Doganaksoy}

NIST/NSF/ASA Associate Research Fellow and General Electric Co.

Schenectady, NY

U.S. DEPARTMENT OF COMMERCE

Technology Administration

National Institute of Standards

and Technology

Center for Computing and Applied Mathematics Statistical Engineering Division

Gaithersburg, MD 20899

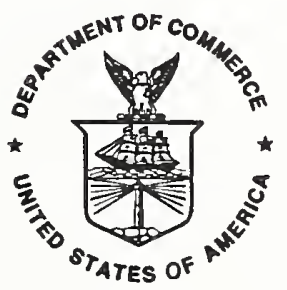

U.S. DEPARTMENT OF COMMERCE Barbara Hackman Franklin, Secretary NATIONAL INSTITUTE OF STANDARDS AND TECHNOLOGY

John W. Lyons, Director 



\section{CONTENTS}

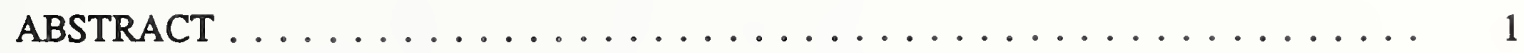

1. INTRODUCTION .............................

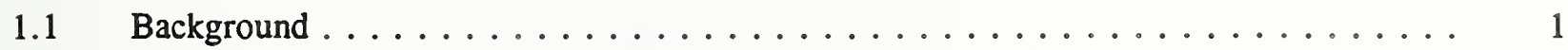

$1.2 \quad$ Example Data . . . . . . . . . . . . . . . . . . . . . . . 2

2. HOW TO RUN THE POWNOR PROGRAM ................... 4

3. THE POWER-NORMAL DISTRIBUTION .................... 9

4. THE POWER-LOGNORMAL DISTRIBUTION . . . . . . . . . . . . . . 13

5. MAXIMUM LIKELIHOOD THEORY FOR FITTING THE POWER-(LOG)NORMAL

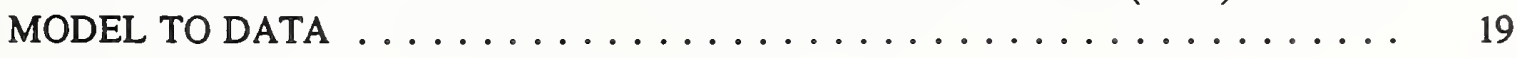

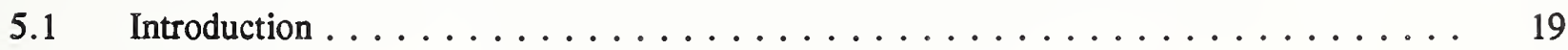

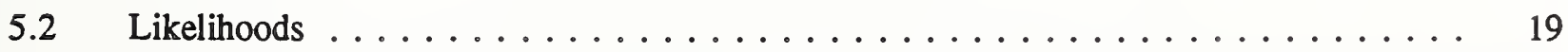

$5.3 \quad$ Maximum Likelihood Estimates . . . . . . . . . . . . . . . . . . . 21

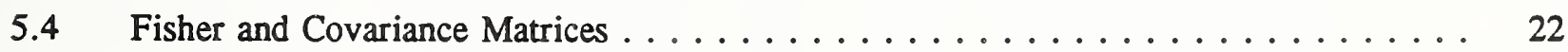

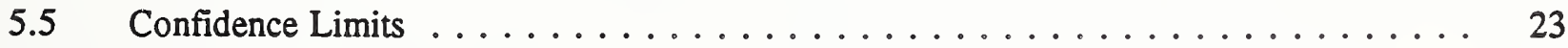

6. NUMERICAL METHODS ......................... 25

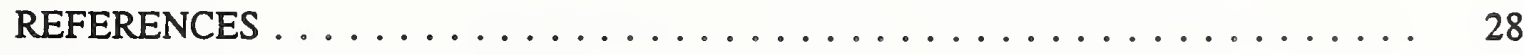





\section{ABSTRACT}

This report presents the power-normal and -lognormal models, which describe the effect of specimen size on the distribution of life or strength of a product or material. Such a model arises when any specimen can be regarded as a series system of smaller portions, where portions of a certain size have a normal or lognormal life (or strength) distribution. Also, this report documents the first computer program that fits this model to data (including censored and interval life data) from specimens of various sizes. The program employs maximum likelihood fitting and provides approximate confidence limits, as well as estimates, for model parameters, distribution percentiles, and other quantities of interest. How to run the program is explained with an analysis of data on time to electromigration failure of aluminum conductors for microcircuits. The program runs on the VAX computer and SUN workstation.

\section{INTRODUCTION}

Purpose. This report describes in detail properties of the power-lognormal and related powernormal models. The report also presents the first computer program for fitting these models to data. This introduction briefly presents the history of the models, the models, the computer program, and an overview of this report. Then the introduction describes electromigration failure data used to illustrate 1) use of the models of Section 3 and 4 and 2) how to run the computer program in Section 2.

\subsection{Background}

History. The power-normal and -lognormal models have long been used to represent the effect of specimen size on the distribution of life or strength of products and materials. In an early application, Chaplin (1880) used the power-normal model to describe the distribution of tensile strength of steel bars of various lengths. Nelson (1990, pp. 385-392) presents models for size effect and applications to cable. Harter (1977) surveys the literature on this and other models for the effect of specimen size for a great variety of applications. Our work on this model and computer program was motivated by an application to data on time to electromigration failure of aluminum conductors in microcircuits. A key electromigration reference is LaCombe and Parks (1986), who use the model for their data.

The models. The power-normal and power-lognormal models are briefly described here. Full details appear in Sections 3 and 4. For specimens of size $S$, the reliability function of the power-normal distribution is the population fraction above the value $y$ (of life, strength, etc.); namely,

$$
\bar{F}\left(y, S ; \mu, \sigma, S_{0}\right)=\{\Phi[-(y-\mu) / \sigma]\}^{S / S_{0}},-\infty<y<\infty .
$$

This is a power of the normal reliability function. Here $\Phi[$ ] is the standard normal cumulative distribution function and the minus sign converts it into a reliability function. The location parameter $\mu$ has the range $-\infty<\mu<\infty$. The scale parameter $\sigma$ is positive. The normal size parameter $S_{0}$ is positive; that is, $y$ has a normal distribution when $S=S_{0}$. In previous work, people had to assume a value for $S_{0}$, which is unknown. The computer program here provides an estimate of $S_{0}$ (and of $\mu$ and $\sigma$ ) from data. The ratio $\rho \equiv S / S_{0}$ is called the power parameter. The reliability function of the power-lognormal distribution is the population fraction above the value $t$; namely, 


$$
\bar{G}\left(t, S ; \mu, \sigma, S_{0}\right)=\{\Phi[-(\ln (t)-\mu) / \sigma]\}^{S / S_{0}}, t>0 .
$$

The parameters $\mu$ and $\sigma$ have the same names and ranges. Here $S_{0}$ is the lognormal size parameter; that is, $t$ has a lognormal distribution when $S=S_{0}$.

Computer program. The computer program POWNOR uses maximum likelihood (ML) methods to fit the model to data. Program output includes ML estimates and approximate confidence limits for model parameters, distribution percentiles, and other quantities of interest. User friendly, the program is written in Fortran 77 and runs on the VAX 11/785 computer under the VMS operating system and on the SUN workstation. It employs IMSL routines. Copies of the program may be requested from the Statistical Engineering Division, Adm. Bldg. A337, National Institute of Standards and Technology, Gaithersburg, MD 20899, (301) 975-2839.

Overview. This report contains:

- Section 2 on how to run the computer program, illustrated with data on time to electromigration failure. Many readers need to read only Section 2.

- Section 3 on the statistical properties of the power-normal model.

- Section 4 on the statistical properties of the related power-lognormal model.

- Section 5 on the maximum likelihood theory for fitting either model to data, which may be censored (unfailed runouts) or interval data.

- Section 6 on the numerical methods employed to carry out the maximum likelihood calculations.

Acknowledgments. The authors gratefully acknowledge the support of this research under their NIST/NSF/ASA Research Fellowship at the National Institute of Standards and Technology (NIST). The National Science Foundation (NSF) generously provided our salary and travel expenses. NIST kindly provided research facilities and colleagues. The American Statistical Association (ASA) handled the Fellowship selection and administration. Dr. Robert Lundegard and Mrs. Ruth Varner of NIST effectively and graciously administered our work at NIST. Mr. Harry Schafft and Dr. Jim Lechner of NIST provided much valued stimulation for this report in our collaboration on electromigration research with them.

\subsection{Example Data}

Overview. This section presents electromigration failure data used to illustrate how to run the program to fit a power-(log)normal model to data. The section first gives background on electromigration. Then it describes the data. Finally it states a purpose of fitting a power-lognormal model to the data. The results of fitting appear in Section 2.

Electromigration. Aluminum conductors in microcircuits can fail from movement of atoms resulting in an open conductor or in a short (due to an aluminum filament growing between adjoining conductors). Such failures occur more quickly at high current density and high temperature. Microcircuit components and conductors are being built smaller and smaller for higher speed, resulting in higher current densities and temperatures in conductors. The microcircuit industry fears that electromigration failure may limit such size reduction. So the industry seeks more knowledge of electromigration including 
a model for the distribution of time to failure and for the effect of conductor length. The powerlognormal model has been proposed, but some experts have observed that such time to failure data for various specimen lengths all appear adequately described by a lognormal distribution. The following data yield information on the adequacy of the power-lognormal and lognormal distributions.

Data. Table 1.1 displays hours to failure of 59 test conductors of length $S=400$ microns. All specimens ran to failure at a certain high temperature and current density. The data come from an interlaboratory comparison reported by Schafft and others (1987). The 59 specimens were all tested under the same temperature and current density. The data appear in a lognormal probability plot in Figure 1.1. The plot is relatively straight. Thus it suggests that a lognormal distribution adequately fits the data and that a power-lognormal distribution fitted to the data is "close" to lognormal; that is, $\rho=S / S_{0}$ is "close" to 1 .

Table 1.1 Hours to Failure of 400-micron Electromigration Specimens

\begin{tabular}{|c|c|c|c|c|c|}
\hline ة. $\equiv \div 5$ & 0.289 & $7 . \equiv 43$ & 5.950 & 6.452 & 5.459 \\
\hline $6 .: 20$ & 4.706 & 8.537 & 2.997 & $8.5 \equiv 1$ & 6.12 \\
\hline 12.033 & 5.361 & 6.958 & 4.283 & 6.522 & 4.12 \\
\hline $7.4 \equiv \Xi$ & 7.495 & 6.573 & 6.533 & 5.530 & 6.08 \\
\hline$\equiv .307$ & б.725 & $8 . \equiv j 2$ & 9.563 & $6.36 \leqq$ & 7.02 \\
\hline 3.336 & $\equiv .228$ & 7.945 & 5.869 & 6.352 & 4.70 \\
\hline $6 . \equiv \div 8$ & 0.254 & $\equiv .009$ & 7.469 & 7.398 & 6.03 \\
\hline $10.0 \leqq 2$ & $7 . \div 96$ & 4.532 & 7.974 & 3.790 & 7.6 \\
\hline$i .224$ & 7.365 & 6.923 & $5.6 \div 0$ & $5.43 \div$ & 7.93 \\
\hline $6 . \equiv 15$ & 6.476 & 6.079 & 10.491 & $\Xi .923$ & \\
\hline
\end{tabular}

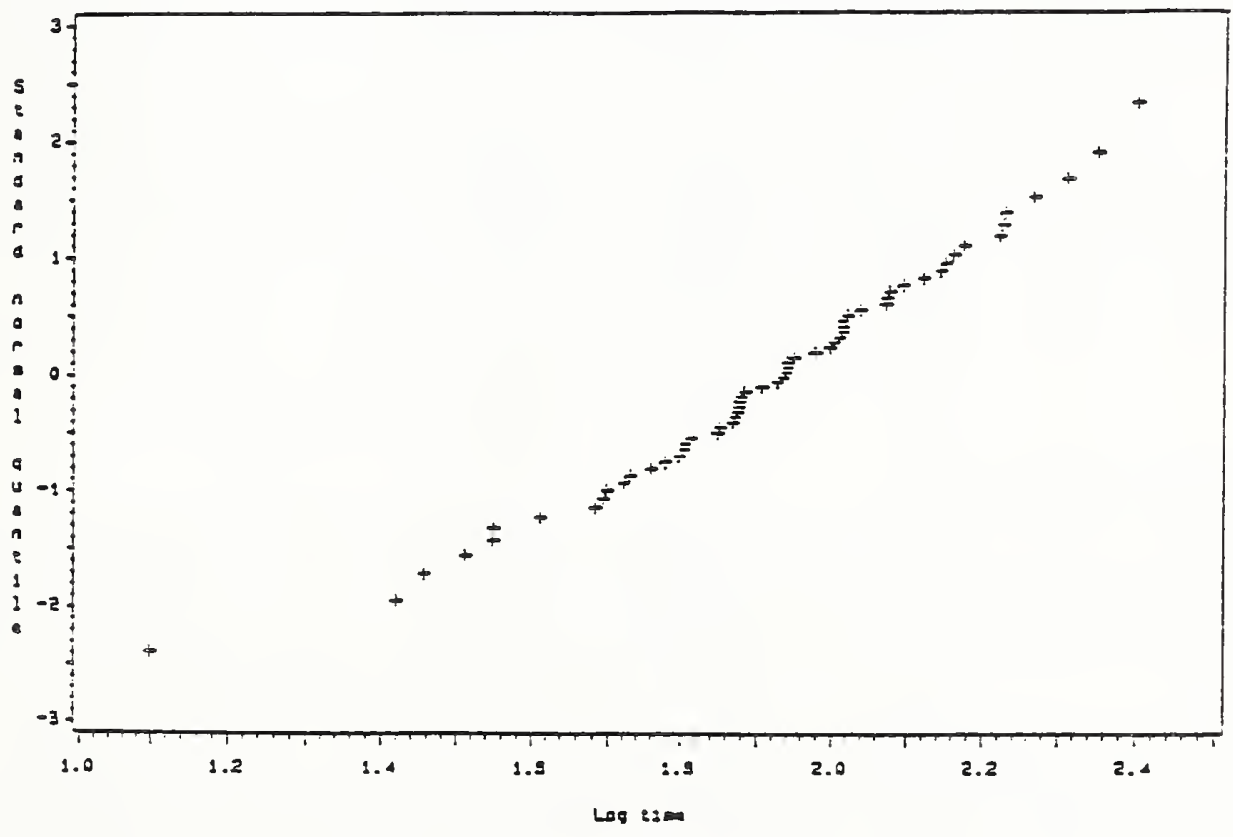

Fig. 1.1 Lognormal Probability plot of 400-micron data. 
Purpose. Fitting the power-lognormal distribution to the data serves certain purposes. First, it provides an estimate of the lognormal length $S_{0}$, which previously has not been properly estimated and may lead to physical insight. Second, wide confidence limits for $\rho=S / S_{0}$ that enclose 1 (the lognormal distribution) would indicate why many data sets appear adequately described by a lognormal distribution. Third, extrapolation to estimate the life distribution of the total length of conductor in microcircuits under normal temperature and current density is of interest. Fourth, the uncertainty of that distribution estimate provides guidance on the appropriate number of test specimens needed to yield estimates of a desired accuracy.

\section{HOW TO RUN THE POWNOR PROGRAM}

Introduction. This section explains how to run the program POWNOR to fit a power-normal or -lognormal model to data. It describes and illustrates input files, program operation, and printed output. The examples here use the electromigration data of Section 1.2.

Input files. The user must create two input files before the program can be run. One of these files POWNOR.DATA stores data on specimen failure times and specimen lengths. The other file POWNOR.OPTIONS contains various user choices (e.g., desired level of detail in printing results, starting values for parameters, etc.). The user can employ his favorite text editor to create the input files. In both of these files, values in a line are separated by one or more spaces (free format). Each line contains at most 80 characters and spaces (typical line length on many computer terminals).

Data file. This file contains data on specimen failure times and specimen lengths. Figure 2.1 shows the electromigration data as they appear in the data file POWNOR.DATA. On each line (record) of this file, enter the data on a particular specimen. The three values on the line for a specimen are:

1. The lower endpoint of the censoring interval. This data value is numerical and must not be below $-1 \mathrm{E} 10$.

2. The upper endpoint of the censoring interval. This data value is numerical and must not exceed 1 E10.

3. Length of the specimen. This data value is numerical and cannot be zero or negative.

Censoring is indicated as follows:

- If the specimen fails at an exactly known age, set the two endpoints of the censoring interval equal to the failure age.

- If the failure is in a known interval, set the lower and upper endpoints equal to the interval endpoints.

- If the specimen is left censored, set the lower endpoint equal to $-1 \mathrm{E} 10$, and the upper value equal to the censoring age.

- If the specimen is right censored, set the lower endpoint equal to the censoring age and the upper endpoint equal to $1 \mathrm{E} 10$. 


\begin{tabular}{|c|c|c|}
\hline LOWER & UPPER & LENGTH \\
\hline 6.545 & 6.545 & 400 \\
\hline 8.120 & 8.120 & 400 \\
\hline 11.038 & 11.038 & 400 \\
\hline 7.459 & 7.459 & 400 \\
\hline 5.807 & 5.807 & 400 \\
\hline 8.336 & 8.336 & 400 \\
\hline 6.948 & 6.948 & 400 \\
\hline 10.092 & 10.092 & 400 \\
\hline 7.224 & 7.224 & 400 \\
\hline 6.515 & 6.515 & 400 \\
\hline 9.289 & 9.289 & 400 \\
\hline 4.706 & 4.706 & 400 \\
\hline 5.381 & 5.381 & 400 \\
\hline 7.495 & 7.495 & 400 \\
\hline 6.725 & 6.725 & 400 \\
\hline 9.218 & 9.218 & 400 \\
\hline 9.254 & 9.254 & 400 \\
\hline 7.496 & 7.496 & 400 \\
\hline 7.365 & 7.365 & 400 \\
\hline 6.476 & 6.476 & 400 \\
\hline 7.543 & 7.543 & 400 \\
\hline 8.687 & 8.687 & 400 \\
\hline 6.958 & 6.958 & 400 \\
\hline 6.573 & 6.573 & 400 \\
\hline 8.532 & 8.532 & 400 \\
\hline 7.945 & 7.945 & 400 \\
\hline 5.009 & 5.009 & 400 \\
\hline 4.531 & 4.531 & 400 \\
\hline 6.923 & 6.923 & 400 \\
\hline 6.071 & 6.071 & 400 \\
\hline 6.956 & 6.956 & 400 \\
\hline 2.997 & 2.997 & 400 \\
\hline 4.288 & 4.288 & 400 \\
\hline 6.538 & 6.538 & 400 \\
\hline 9.663 & 9.663 & 400 \\
\hline 6.869 & 6.869 & 400 \\
\hline 7.489 & 7.489 & 400 \\
\hline 7.974 & 7.974 & 400 \\
\hline 5.640 & 5.640 & 400 \\
\hline 10.491 & 10.491 & 400 \\
\hline 6.492 & 6.492 & 400 \\
\hline 8.591 & 8.591 & 400 \\
\hline 6.522 & 6.522 & 400 \\
\hline 5.589 & 5.589 & 400 \\
\hline 6.369 & 6.369 & 400 \\
\hline 6.352 & 6.352 & 400 \\
\hline 7.398 & 7.398 & 400 \\
\hline 8.799 & 8.799 & 400 \\
\hline 5.434 & 5.434 & 400 \\
\hline 5.923 & 5.923 & 400 \\
\hline 5.459 & 5.459 & 400 \\
\hline 6.129 & 6.129 & 400 \\
\hline 4.137 & 4.137 & 400 \\
\hline 6.087 & 6.087 & 400 \\
\hline 7.024 & 7.024 & 400 \\
\hline 4.700 & 4.700 & 400 \\
\hline 6.033 & 6.033 & 400 \\
\hline 7.683 & 7.683 & 400 \\
\hline 7.937 & 7.937 & 400 \\
\hline
\end{tabular}

Fig. 2.1 Electromigration life data in the file POWNOR.DATA. 
Section 5.2 defines different types of censored data. The electromigration data in Figure 2.1 do not include any censored observations. In this example, each specimen has the same length, 400 microns. The file must not contain the headings LOWER, UPPER, and LENGTH added to Figure 2.1 for explanation. The data file cannot contain more than 10,000 lines.

Options file. The POWNOR.OPTIONS file contains the following choices of the user, which are explained later:

- Whether or not log transformation of failure data in POWNOR.DATA is required.

- Level of detail in documenting the iteration history.

- $\quad$ Starting values for parameters to be estimated and fixed parameter values.

- Specimen lengths for which estimates of distribution percentiles and their associated confidence limits are desired.

Figure 2.2 shows the POWNOR.OPTIONS file used for the electromigration data example.

$\begin{array}{lr}1 & \\ 1 & \\ 0 & 2.9219 \\ 0 & -0.7186 \\ 0 & 2.5415 \\ 400 & \end{array}$

Fig. 2.2 The POWNOR.OPTIONS file with options for the electromigration data.

Log transformation. Enter 1 on the first line to calculate natural logs of the failure (and censoring) times in POWNOR.DATA. If no $\log$ transformation is required, enter 0 . The electromigration example requires $\log$ transformation of failure times. This is provided by the value 1 on the first line of the file POWNOR.OPTIONS in Figure 2.2. The program fits the power-normal distribution to the log data.

Iteration history. Enter 1 on the second line for detailed printout of iterations. The program then stores the iterations output in a file named POWNOR.ITERATIONS. Enter 0, if the detailed iteration history is not required. This output is recommended as an aid to understanding iterative fitting that is not converging well. The POWNOR.OPTIONS file in Figure 2.2 requests that the detailed iterations be saved.

Parameter values. The user is required to supply starting values of the parameters for numerical maximization of the sample log likelihood function. Section 6 provides guidelines to obtain starting values. Alternatively, the user may wish to hold some of the parameters fixed at desired values. The lines 3,4 , and 5 of POWNOR.OPTIONS specify such choices for $\mu, \ln (\sigma)$, and $\ln \left(S_{0}\right)$, respectively. Each of these lines must contain 2 values. A 0 for the first value indicates that the parameter is to be estimated from data. The second value is then taken to be the starting value for the parameter. A 1 for the first value indicates that the parameter value is held fixed at the second value. This feature is particularly 
useful in likelihood ratio tests for parameters, as described in Nelson (1990, Chapter 9). Figure 2.2 indicates that all three parameters in the electromigration data example are to be estimated from data. The starting values for the $\mu, \ln (\sigma)$, and $\ln \left(S_{0}\right)$ are $2.9219,-0.7186$, and 2.5415 , respectively. These starting values were obtained following the guidelines in Section 6.

Specimen lengths for percentile estimates. Line 6 and the ones below it contain the specimen lengths for which estimates and confidence limits for distribution percentiles are desired. Each line contains a single length value. The user can specify at most 10 lengths. In the electromigration example, such percentile estimates are requested only for specimens of length 400 microns.

Program operation. The user must previously create the two input files as described above. The program then can be run by typing RUN POWNOR at the \$ prompt of a VAX computer with a VMS operating system. Figure 2.3 shows program messages during run for the electromigration data example. The underlined entries in the figure are user responses.

\section{\$ RUN POWNOR}

PROGRAM MESSAGE: The program POWNOR reads the user options from POWNOR. OPTIONS and data lines from POWNOR.DATA

PROGRAM MESSAGE: Iteration history is in POWNOR.ITERATIONS

PROGRAM MESSAGE: Program output is in POWNOR.OUTPUT

FORTRAN STOP

\section{\$ TYPE POWNOR.OUTPUT}

Fig. 2.3 How to submit a POWNOR run on a VAX computer.

Output. Figure 2.4 displays the program output in the file POWNOR.OUTPUT from the ML fitting of a power-normal model to the logs of the electromigration data. This output is explained below; the numbers correspond to those in the figure.

I. This output states the number of uncensored and censored observations in the data file POWNOR.DATA.

II. This output shows the ML estimates of the (transformed) model parameters $\left(\mu, \ln \sigma, \ln S_{0}\right)$ and their approximate $95 \%$ confidence limits. For example, the estimate of $\sigma$ is $\hat{\sigma}=\exp (-0.7336)=$ 0.48 , and its $95 \%$ confidence limits are $\sigma=\exp (-1.6375)=0.19$ and $\tilde{\sigma}=\exp (0.1704)=$ 1.19 . 
Developed by Necip Doganaksoy and Wayne Nelson under 1991 Fellowship grant from ASA/NSF/NIST. The program is documented in NIST Report\#.

I: Total number of data cases $=59$ Exact observations $=59$ Left censored observations $=0$ $\begin{array}{ll}\text { Right censored observations }= & 0 \\ \text { Interval censored observations }= & 0\end{array}$

Maximized log-likelihood $=55.8554$

Maximum likelihood estimates for distribution parameters with approximate $95 \%$ confidence limits

$\begin{array}{ll} & \text { Parameter } \\ & 1 \mathrm{mu} \\ 2 & \ln (\text { signa) } \\ & 3 \ln (\text { normal length) }\end{array}$
ML Estimate 2.8779 $-0.7336$
2.6779

Lower Limit

0.8280

$-1.6375$

$-3.1808$
Upper Limit

4.9279

0.1704

8.5366
Std. Error 1.0459 0.4612 2.9891

III:

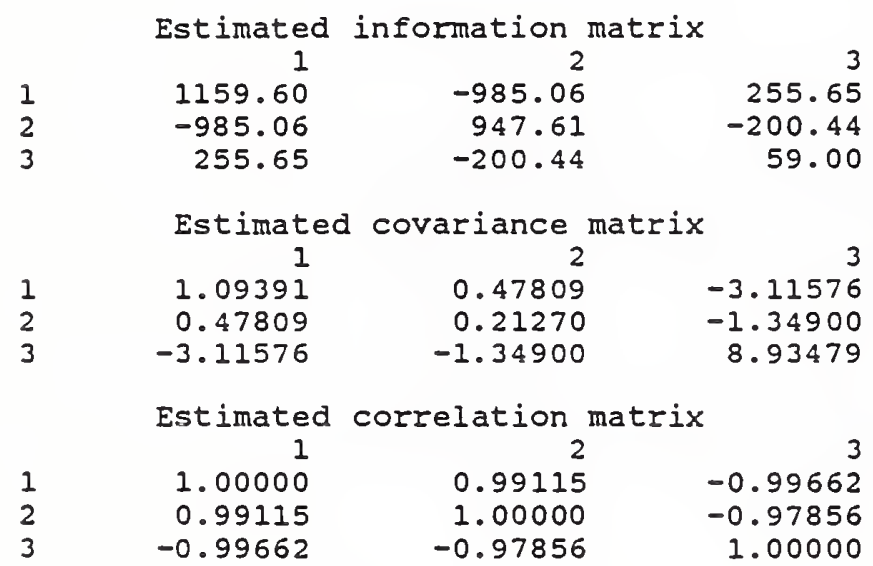

IV: Maximum likelihood estimates for distribution percentiles with approximate $95 \%$ confidence limits

Length $=$

400.0000

$\begin{array}{lrr}\text { Pct. } & \text { ML Estimate } & \text { Lower Limit } \\ 0.1 & 0.9731 & 0.5994 \\ 0.2 & 1.0541 & 0.7256 \\ 0.5 & 1.1664 & 0.8968 \\ 1 & 1.2561 & 1.0297 \\ 2 & 1.3507 & 1.1655 \\ 5 & 1.4853 & 1.3496 \\ 10 & 1.5973 & 1.4930 \\ 30 & 1.8074 & 1.7357 \\ 50 & 1.9360 & 1.8719 \\ 70 & 2.0528 & 1.9921 \\ 90 & 2.2044 & 2.1380 \\ 95 & 2.2714 & 2.1944 \\ 98 & 2.3431 & 2.2475 \\ 99 & 2.3890 & 2.2776 \\ 99.5 & 2.4298 & 2.3020 \\ 99.9 & 2.5108 & 2.3439\end{array}$

Upper Limit
1.3468
1.3825
1.4360
1.4825
1.5358
1.6210
1.7016
1.8792
2.0001
2.1135
2.2709
2.3485
2.4387
2.5004
2.5576
2.6776

std. Error 0.1907 0.1676 0.1375

0.1155

0.0945

0.0692

0.0532

0.0366

0.0327

0.0310

0.0339

0.0393

0.0488

0.0568

0.0652

0.0851

Fig. 2.4 POWNOR output for the electromigration data. 
III. The local estimates of the information, covariance, and correlation matrices for the (transformed) parameter estimates are shown here. The high correlations (near \pm 1 ) indicate that convergence to the ML estimates may be incomplete and that a better parameterization of the model could avoid convergence problems.

IV. This output shows the ML estimates of percentiles (log values) and their $95 \%$ confidence limits at each specified length.

The theory underlying the ML fitting is in Section 5. Section 6 explains numerical methods used in fitting.

Reparametrization. The program POWNOR maximizes the log likelihood function with respect to $\mu, \ln (\sigma)$, and $\ln \left(S_{0}\right)$. Sometimes, this parametrization of the model must be altered to improve numerical convergence to the ML estimates. Linear orthogonalization of the parameters provides a convenient means for improving convergence as described, for example, in Ross (1990). Section 6 describes such linear orthogonalization of parameters. Another version of the program POWNOR_ORTHO uses such orthogonalization. The input files, operation, and outputs of POWNOR_ORTHO are very similar to those of POWNOR.

\section{THE POWER-NORMAL DISTRIBUTION}

Introduction. This section presents properties of the power-normal distribution. As background, readers need to know basic properties of the normal distribution and normal probability paper. For example, Nelson $(1982,1990)$ covers these topics and the series-system model (below) in detail. The following topics include its reliability function, cumulative distribution function, the series-system model, percentiles, median, probability density, mode, mean, variance, standard deviation, and hazard function (failure rate).

Reliability function and cdf. The reliability function of the power-normal distribution is the population fraction above the value $y$; namely,

$$
\bar{F}(y ; \mu, \sigma, \rho) \equiv\{\Phi[-(y-\mu) / \sigma]\}^{\rho},-\infty<y<\infty .
$$

Here $-\infty<\mu<\infty$ is the location parameter, $\sigma>0$ is the scale parameter, and $\rho>0$ is the power parameter $\rho=S / S_{0}$ in (1.1). Note the minus sign in the standard normal cumulative distribution function $\Phi[$ ]; it yields the upper tail probability of the normal distribution. For $\rho=1$, the distribution is normal; only then is $\mu$ the mean and median of the distribution, and only then is $\sigma$ the standard deviation of the distribution. The cumulative distribution function (cdf) is the population fraction below $y$; namely,

$$
F(y ; \mu, \sigma, \rho)=1-\{\Phi[-(y-\mu) / \sigma]\}^{\rho},-\infty<y<\infty .
$$

The distribution is simpler in terms of the standardized deviate $z \equiv(y-\mu) / \sigma$. Then

$$
\left.\bar{F}(y ; \mu, \sigma, \rho)=[\Phi(-z)]^{\rho}, F(y ; \mu, \sigma, \rho)\right]=1-[\Phi(-z)]^{\rho},-\infty<z<\infty .
$$

David (1981, p287) lists tables of these functions for integer $\rho$ values. 


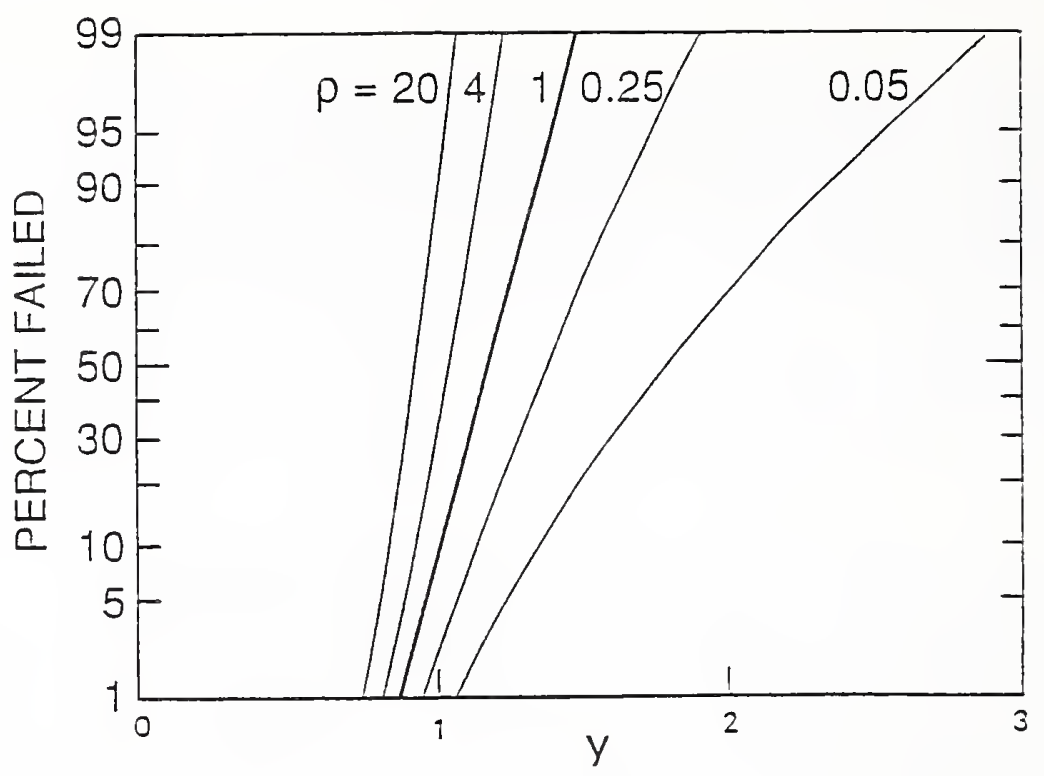

Fig. 3.1 Power-normal cdfs on normal probability paper.

Probability plot. Figure 3.1 displays power-normal cdfs for selected $\rho$ values on normal probability paper. For $\rho=1$, the cdf is normal and is a straight line. For $\rho>1$, the cdfs curve concave to the left and shift toward lower $y$ (and $z$ ) values as $\rho$ gets larger. For $0<\rho<1$, the cdfs curve concave to the right and shift toward higher $y$ (and $z$ ) values as $\rho$ gets smaller.

Approximate cdf. For large $\rho$, the exact cdf formula (3.2) requires a table or algorithm for $\Phi(\quad)$ that is accurate in the far tails. Asymptotically as $\rho \rightarrow \infty$, the power-normal cdf approaches the cdf of a smallest extreme value distribution (convergence in law or in distribution). Bury (1975) gives the location and scale parameters of that distribution as a function of $\mu, \sigma$, and $\rho$. Convergence to this asymptotic distribution is slow. That is, $\rho$ must be very large (say well above 100 ) for a satisfactory approximation.

Series-system model. In some applications, product comes in different sizes, and its life, strength, or some property depends on size. For example, time to electromigration failure of conductors in microcircuitry depends on conductor length. Also, strength of metal bars depends on bar length (Chaplin (1880)). Harter (1977) surveys models for the effect of size. The following series-system model is a simple, widely-used model for the effect of size. For purposes of presenting the model, reliability terminology and product life are used. The assumptions of the series-system model for size effect are:

1. If product is conceptually divided into portions of any size of interest, it is assumed that the times to failure of the portions are statistically independent.

2. The product fails when the first such portion fails.

3. Portions of size $S_{0}$ have a reliability function $R_{0}(t)$, which is the population fraction surviving beyond age $t$. 
It follows from these assumptions that product of size $S$ has a reliability function

$$
R_{S}(t)=\left[R_{0}(t)\right]^{S / S_{O}}
$$

Nelson $(1982,1990)$ presents this model in more detail and gives various applications. When $R_{0}(t)$ is the reliability function of a normal distribution, (3.4) is the power-normal reliability function (3.1). Then the power parameter $\rho \equiv S / S_{0}$ is the ratio of the product size $S$ to the "normal size" $S_{0}$.

Fractiles. The $F$ fractile of the power-normal distribution is

$$
\eta_{F}=\mu+z_{F} \cdot \sigma
$$

here $z_{F}$, is the $F^{\prime}=1-(1-F)^{1 / \rho}$ fractile of the standard normal distribution. For $\rho>>1, F^{\prime}$ usually is very near zero. For $\rho$ near zero, $F^{\prime}$ usually is very near one. Tables of $z_{F^{\prime}}$ appear in most statistics books. However, few tables adequately provide $z_{F}$, values for $F^{\prime}$ very near zero or one.

Median. The median is the 0.50 fractile $\eta_{0.50}$. It is a "center" of the distribution in that half of the population is below $\eta .50$ and half is above it. For the power-normal distribution,

$$
\eta_{0.50}=\mu+z_{F} . \sigma
$$

here $F^{\prime}=1-(0.5)^{1 / \rho}$. For $\rho=1$, the distribution is normal, $F=0.50, z_{F}=0$, and $\eta_{0.50}=\mu$.

Density. The power-normal probability density is the derivative of the cdf (3.2); namely,

$$
f(y ; \mu, \sigma, \rho)=(\rho / \sigma) \phi[(y-\mu) / \sigma]\{\Phi[-(y-\mu) / \sigma]\}^{\rho-1} \text {; }
$$

here $\phi(z) \equiv(2 \pi)^{-1 / 2} \exp \left(-z^{2} / 2\right)$ is the standard normal probability density. In terms of the standardized deviate $z=(y-\mu) / \sigma$,

$$
f(y ; \mu, \sigma, \rho)=(\rho / \sigma) \phi(z)[\Phi(-z)]^{\rho-1} .
$$

Figure 3.2 displays such probability densities as a function of $z$ for selected $\rho$ values where $\mu=0$ and $\sigma=1$. For $\rho=1$, the density is the standard normal density. These densities are unimodal.

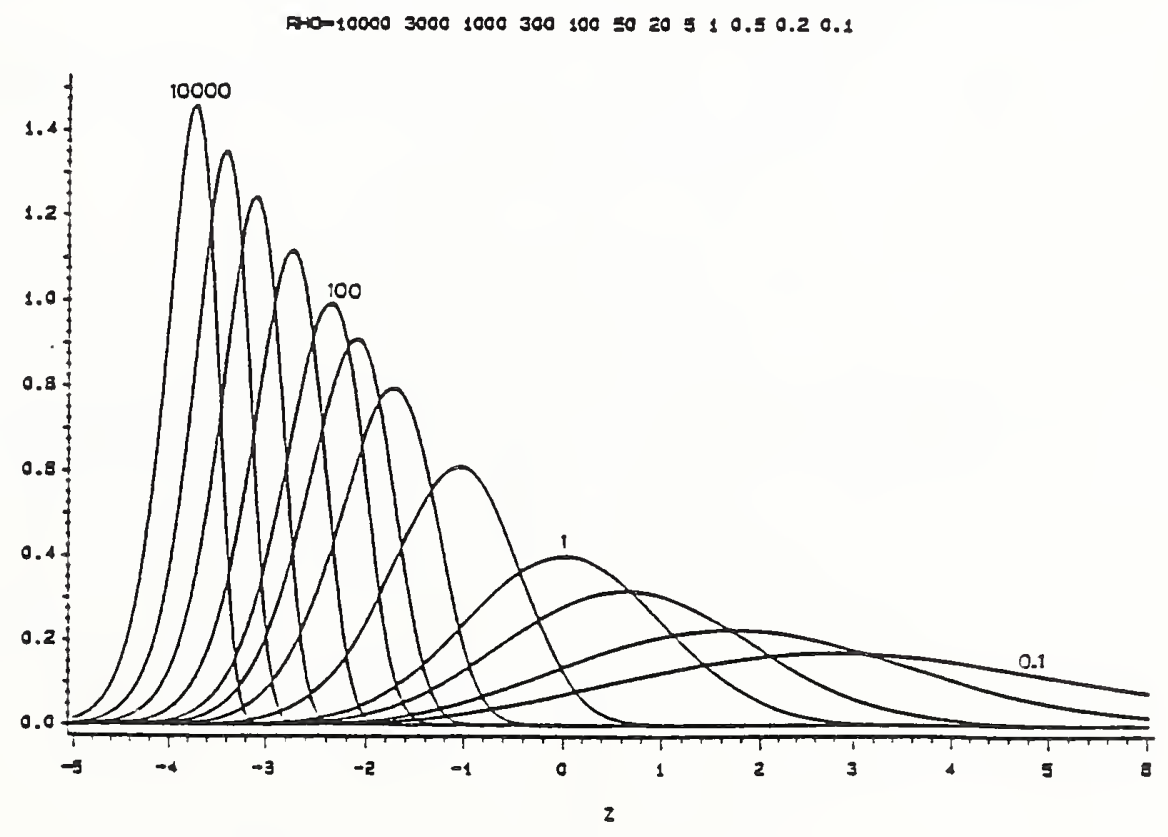

Fig. 3.2 Power-normal probability densities. 
Mode. The mode of a power-normal distribution is the value $\eta_{m}$ where the probability density peaks. It is the most probable $y$ value. $\eta_{m}$ is the solution of $d f(y ; \mu, \sigma, \rho) / d y=0$; namely,

$$
\eta_{m}=\mu+z_{m} \sigma \text {. }
$$

Here $z_{m}$ is a function of just $\rho$ and is the solution of $-z_{m} \Phi\left(-z_{m}\right) / \phi\left(z_{m}\right)=\rho-1$. For $\rho=1, z_{m}=0$ and the mode $\eta_{m}=\mu$, a well-known property of the normal distribution.

Mean. The mean of the power-normal distribution is

$$
E Y \equiv \int_{-\infty}^{\infty} y f(y ; \mu, \sigma, \rho) d y=\mu+E_{\rho} \sigma .
$$

Here the mean $E_{\rho}$ for the standardized distribution ( $\mu=0$ and $\left.\sigma=1\right)$ is a function of $\rho$ only; namely,

$$
E_{\rho} \equiv \int_{-\infty}^{\infty} z \rho \phi(z)[\Phi(-z)]^{\rho-1} d z
$$

For integer $\rho, E_{\rho}$ is the mean of the first order statistic of a sample of size $\rho$. David $(1981, \mathrm{p} 291)$ lists tables of $E_{\rho}$ for integer $\rho$. $E_{\rho}$ apparently has not been tabulated for non-integer $\rho$; then (3.11) must be evaluated by numerical quadrature.

Variance and standard deviation. The variance of the power-normal distribution is

$$
V(Y) \equiv \int_{-\infty}^{\infty}(y-E Y)^{2} f(y ; \mu, \sigma, \rho) d y=V_{\rho} \sigma^{2}
$$

here the variance $V_{\rho}$ for the standardized distribution $(\mu=0$ and $\sigma=1)$ is

$$
V_{\rho} \equiv \int_{-\infty}^{\infty}\left(z-E_{\rho}\right)^{2} \rho \phi(z)[\Phi(-z)]^{\rho-1} d z
$$

For integer $\rho, V_{\rho}$ is the variance of the first order statistic of a sample of size $\rho$ from a standard normal distribution. David $(1981, \mathrm{p} 291)$ lists tables of $V_{\rho}$ for integer $\rho . V_{\rho}$ apparently has not been tabulated for non-integer $\rho$; then (3.13) must be evaluated by numerical quadrature. The standard deviation of the power-normal distribution is

$$
s d(Y)=[V(Y)]^{1 / 2}=V_{\rho}^{1 / 2} \sigma .
$$

For $\rho=1$, the distribution is normal, and $V_{1}=1, V(Y)=\sigma^{2}$, and $s d(Y)=\sigma$.

Hazard function. Much used in reliability work, the hazard function (also called the instantaneous failure rate) of the power-normal distribution is

$$
\begin{aligned}
h(y ; \mu, \sigma, \rho) & \equiv f(y ; \mu, \sigma, \rho) /[1-F(y ; \mu, \sigma, \rho)] \\
& =(\rho / \sigma) \phi[(y-\mu) / \sigma] / \Phi[-(y-\mu) / \sigma] \\
& =\rho \cdot h[(y-\mu) / \sigma]=\rho \cdot h(z) .
\end{aligned}
$$

Here $h(z) \equiv \phi(z) / \Phi(-z)$ is the hazard function of the standard normal distribution and is an increasing function of $z=(y-\mu) / \sigma$. Figure 3.3 depicts $h(z)$. Any power-normal hazard function is merely a multiple $\rho$ of $h(z)$. 


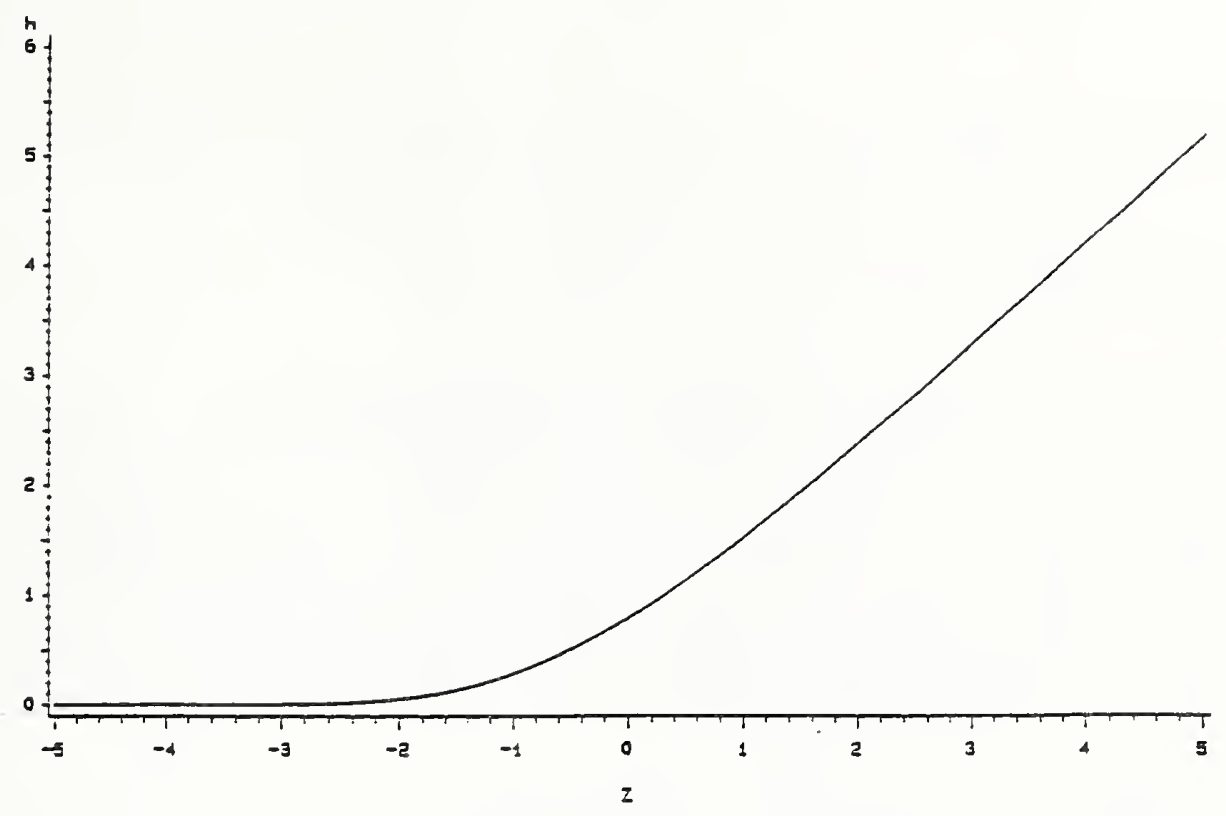

Fig. 3.3 Standard power-normal hazard function.

\section{THE POWER-LOGNORMAL DISTRIBUTION}

Introduction. This section presents properties of the power-lognormal distribution. As background, readers need to know basic properties of the power-normal and normal distributions and lognormal probability paper. For example, Nelson $(1982,1990)$ covers these topics and the series-system model (below) in detail. The following topics include its reliability and cumulative distribution functions, its relationship to the power-normal distribution, the series-system model, percentiles, median, probability density, mode, mean, variance, standard deviation, and hazard function (failure rate).

Reliability function. The reliability function of the power-lognormal distribution is the population fraction surviving beyond age $t>0$; namely,

$$
\bar{G}(t ; \mu, \sigma, \rho) \equiv\{\Phi[-(\ln (t)-\mu) / \sigma]\}^{\rho}, t>0 .
$$

Here $\Phi[$ ] is the standard normal cumulative distribution function, and $\rho>0$ is called the power parameter. Also $\mu(-\infty<\mu<\infty)$ is called the location parameter, but is better called "mu". Strictly speaking, $\mu$ is a location parameter only for the power-normal distribution when $y=\ln (t)$ is used. For the power-lognormal distribution, $\tau=e^{\mu}$ is a scale parameter as shown below. Similarly, $\sigma>0$ is called the scale parameter, but is better called "sigma". Strictly speaking, $\sigma$ is a scale parameter only for the power-normal distribution, when $y=\ln (t)$ is used. For the power-lognormal distribution, $\rho$ and $\sigma$ are actually shape parameters. For $\rho=1$, the distribution is lognormal; only then is $\mu$ the mean of $\log$ life $y=\ln (t)$, is $e^{\mu}$ the median life, and is $\sigma$ the standard deviation of $\log$ life $y=\ln (t)$. The lognormal distribution is briefly presented in Nelson $(1982,1990)$ and in many other statistics books; Shimizu and Crow (1988) devote a book to it. namely,

Cdf. The cumulative distribution function (cdf) is the population fraction failing before age $t$;

$$
G(t ; \mu, \sigma, \rho)=1-\{\Phi[-(\ln (t)-\mu) / \sigma]\}^{\rho}, t>0
$$


The distribution is simpler in terms of the log-transformed standardized deviate $z \equiv[\ln (t)-\mu] / \sigma$. Then

$$
\bar{G}(t ; \mu, \sigma, \rho)=[\Phi(-z)]^{\rho}, \quad G(t ; \mu, \sigma, \rho)=1-[\Phi(-z)]^{\rho} .
$$

David (1981, p287) list tables of these functions for integer $\rho$ values.

Probability plot. Figure 4.1 displays power-lognormal cdfs for selected $\rho$ values on lognormal probability paper. Figure 4.1 is the same as Figure 3.1, except that Figure 4.1 has a data scale (for $t$ ) that is logarithmic and Figure 3.1 has a data scale (for $y$ ) that is linear. For $\rho=1$, the cdf is lognormal and is a straight line. For $\rho>1$, the cdfs curve concave to the left and shift toward lower $t$ (and $z$ ) values as $\rho$ gets larger. For $0<\rho<1$, the cdfs are curves concave to the right and shift toward higher $t$ (and $z$ ) values as $\rho$ gets smaller. Figure 4.2 shows selected cdfs that have common values for the 0.25 and 0.75 fractiles to give these distributions roughly the same spread, as if fitted to the same data set. The figure shows that the lower tails differ appreciably. Thus it is important to know $\rho$ if extrapolating into the lower tail in high reliability applications.

Other parameters. For some purposes, it is useful to express the distribution in terms of its true scale parameter $\tau \equiv \exp (\mu)$. Then

$$
\begin{gathered}
\bar{G}(t ; \mu, \sigma, \rho)=\{\Phi[-(\ln (t / \tau)) / \sigma]\}^{\rho}=\left\{\Phi\left[-\left(\ln (t / \tau)^{1 / \sigma}\right)\right]\right\}^{\rho}, \\
G(t ; \mu, \sigma, \rho)=1-\{\Phi[-(\ln (t / \tau)) / \sigma]\}^{\rho}=1-\left\{\Phi\left[-\left(\ln (t / \tau)^{1 / \sigma}\right)\right]\right\}^{\rho} .
\end{gathered}
$$

For $\rho=1, \tau$ is the distribution median; otherwise, it is the $1-(0.5)^{\rho}$ fractile.

Approximate cdf. For large $\rho$, the exact cdf formula (4.2) requires a table or algorithm for $\Phi($ ) that is accurate in the far tails. Asymptotically as $\rho \rightarrow \infty$, the power-lognormal cdf approaches the cdf of a Weibull distribution (convergence in law or in distribution). Bury (1975) gives the scale and shape parameters of that Weibull distribution as a function of $\mu, \sigma$, and $\rho$. Convergence to this asymptotic Weibull distribution is slow. That is, $\rho$ must be very large (say, well above 100) for a satisfactory approximation.

Series-system model. As described in Section 3, a product may come in different sizes, and its life may depend on size. The previous assumptions for a series-system model are assumed here. However, if in (3.4) the reliability function $R_{0}(t)$ for product of size $S_{0}$ is lognormal, then the reliability function (3.4) of product of size $S$ is the power-lognormal one (4.1). Then the power parameter $\rho=S / S_{0}$ is the ratio of the product size $S$ to the "lognormal size" $S_{0}$.

Relation to the power-normal. The power-lognormal distribution has a relationship to the simpler power-normal distribution. If $t$ has a power-lognormal distribution with parameters $\mu, \sigma$, and $\rho$, then $y=\ln (t)$ has a power-normal distribution with the same values of the parameters $\mu, \sigma$, and $\rho$. Thus, one can analyze power-lognormal data by fitting the power-normal distribution to the log data $y=\ln (t)$. The power-normal distribution is simpler in that only $\rho$ is a shape parameter, whereas, for the powerlognormal distribution, both $\rho$ and $\sigma$ are shape parameters. 


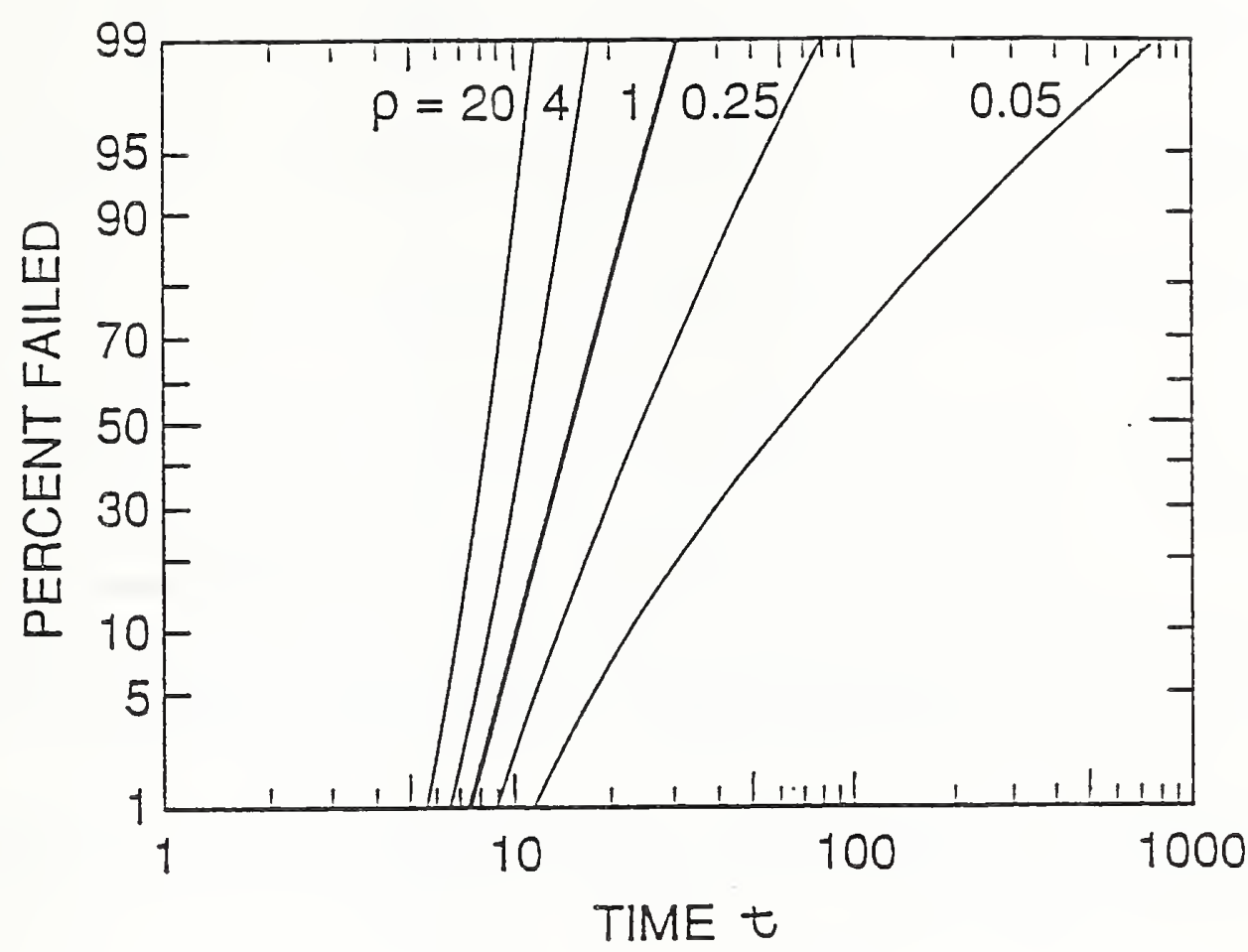

Fig. 4.1 Power-lognormal cdfs on lognormal probability paper.

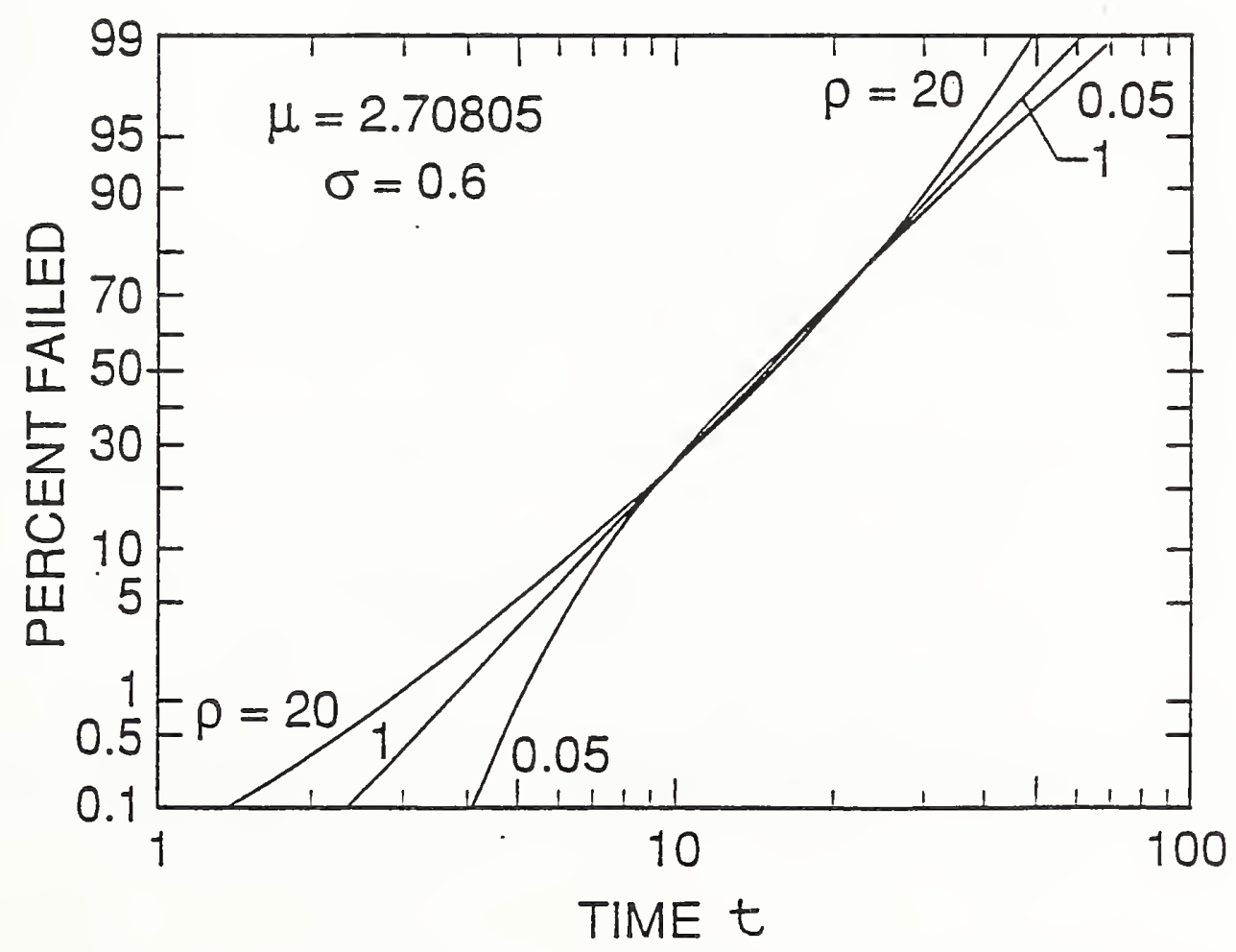

Fig. 4.2 Power-lognormal cdfs with common 0.25 and 0.75 fractiles. 
Fractiles. The $F$ fractile of the power-lognormal distribution is

$$
t_{F}=\exp \left(\mu+z_{F}, \sigma\right)
$$

here $z_{F}$ is the $F^{\prime}=1-(1-F)^{1 / \rho}$ fractile of the standard normal distribution. $t_{F}$ is the antilog of the $F$ fractile (3.5) of the corresponding power-normal distribution.

Median. The median of the power-lognormal distribution is

$$
t_{0.50}=\exp \left(\mu+z_{F}, \sigma\right)
$$

where $F^{\prime}=1-(0.5)^{1 / \rho}$. For $\rho=1$ (the lognormal distribution), $z_{F^{\prime}}=0$ and $t_{0.50}=\exp (\mu)$.

Density. The power-lognormal probability density is the derivative of the cdf (4.2); namely,

$$
g(t ; \mu, \sigma, \rho)=(\rho / t \sigma) \phi\{[\ln (t)-\mu] / \sigma\}[\Phi\{-[\ln (t)-\mu] / \sigma\}]^{\rho-1} ;
$$

here $\phi(z) \equiv(2 \pi)^{-1 / 2} \exp \left(-z^{2} / 2\right)$ is the standard normal probability density. Figure 4.3 displays such densities as a function of $r=t / \tau$, where $\tau=\exp (\mu)$. The densities have a variety of shapes depending on the $\rho$ and $\sigma$ values. These densities are unimodal. For $\rho=1$, the density is lognormal.

Mode. The mode of a power-lognormal distribution is the value $t_{m}$ where the probability density peaks. It is the most probable value. $t_{m}$ is the solution of $d g(t ; \mu, \sigma, \rho) / d t=0$; namely, the solution $z_{m} \equiv\left[\ln \left(t_{m}\right)-\mu\right] / \sigma$ of

$$
\left(z_{m}+\sigma\right) \Phi\left(-z_{m}\right)=-(\rho-1) .
$$

Here $z_{m}$ is a function of $\rho$ and $\sigma$. For $\rho=1$ (the lognormal distribution), $z_{m}=-\sigma$ and $t_{m}=\exp \left(\mu-\sigma^{2}\right)$.

Mean. The mean of the power-lognormal distribution is

$$
\begin{aligned}
E T & \equiv \int_{0}^{\infty} t g(t ; \mu, \sigma, \rho) d t \\
& =\int_{0}^{\infty}(\rho / \sigma) \phi\{[\ln (t)-\mu] / \sigma\}[\Phi\{-[\ln (t)-\mu] / \sigma\}]^{\rho-1} d t . \\
& =\tau \int_{-\infty}^{\infty} \rho e^{\mu \sigma} \phi(u)\{\Phi(-u)\}^{\rho-1} d u \equiv \tau E(\sigma, \rho) .
\end{aligned}
$$

Here $\tau=\exp (\mu)$ is the scale parameter, and $E(\sigma, \rho)$ is a function of $\sigma$ and $\rho$ but not $\mu=\ln (\tau)$. David (1981, p 288) references tables of $E(\sigma, \rho)$ for integer $\rho$ and $\sigma=1$. The integral can be evaluated with numerical quadrature.

Variance and standard deviation. The variance of the power-lognormal distribution is

$$
\begin{aligned}
V(T) & \equiv \int_{0}^{\infty}(t-E T)^{2} g(t ; \mu, \sigma, \rho) d t \\
& =\int_{0}^{\infty}(t-E T)^{2}(\rho / \sigma t) \phi\{[\ln (t)-\mu] / \sigma\}[\Phi\{-[\ln (t)-\mu] / \sigma\}]^{\rho-1} d t, \\
& =\tau^{2} \int_{-\infty}^{\infty}\left[e^{\sigma u}-E(\sigma, \rho)\right]^{2} \rho \phi(u)[\Phi(-u)]^{\rho-1} d u \equiv \tau^{2} V(\sigma, \rho) .
\end{aligned}
$$

Here $\tau=\exp (\mu)$ is the scale parameter, and $V(\sigma, \rho)$ is a function of $\sigma$ and $\rho$ but not $\mu=\ln (\tau)$. David (1981, p288) references tables of the integral for integer $\rho$ and $\sigma=1$. The integral can be evaluated with numerical quadrature. The standard deviation of the power-lognormal distribution is 

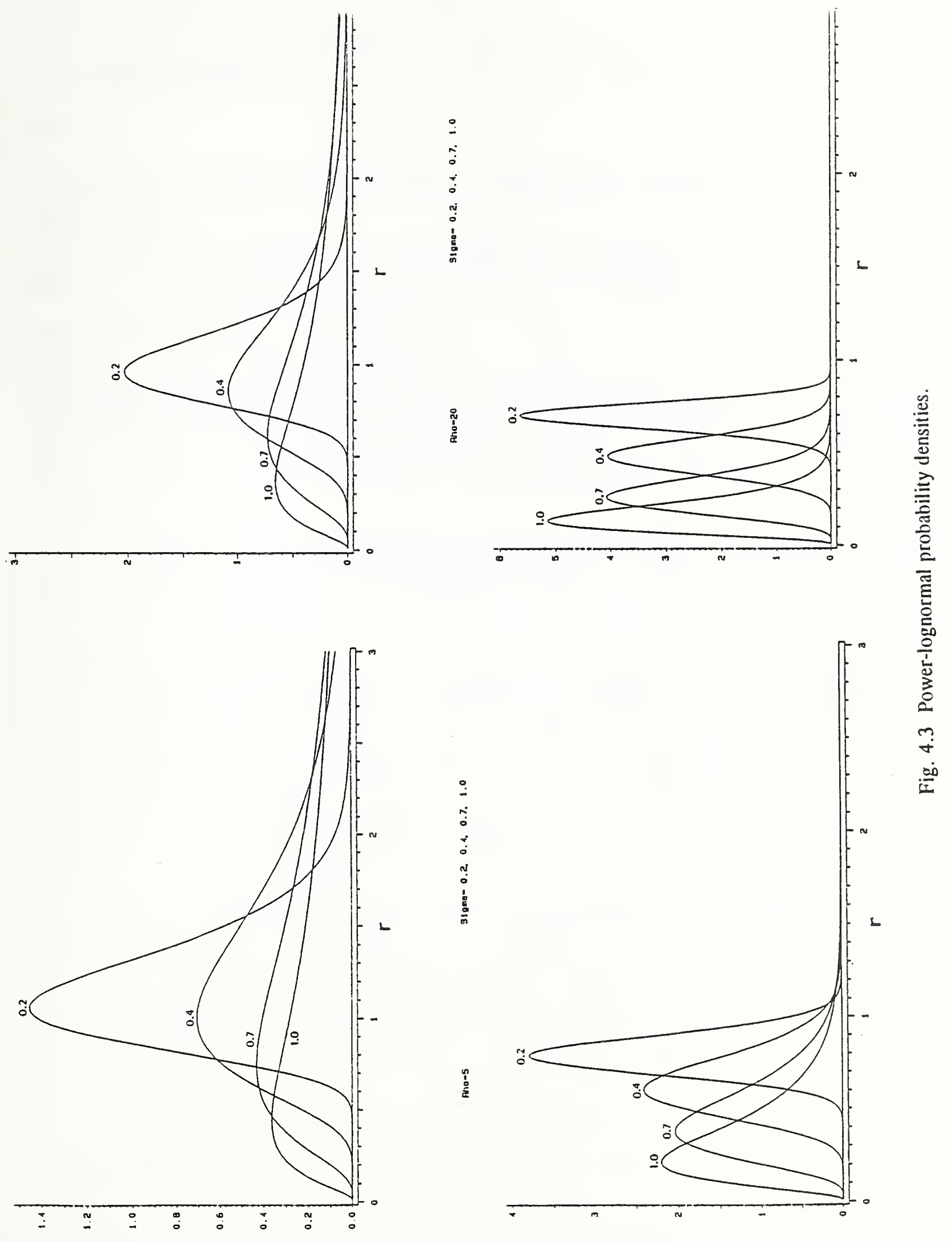


$$
s d(T) \equiv[V(T)]^{1 / 2}=\tau[V(\sigma, \rho)]^{1 / 2} .
$$

For $\rho=1$, the lognormal distribution, $V(\sigma, 1)=\left[\exp \left(\sigma^{2}\right)-1\right] \exp \left(\sigma^{2}\right), V(T)=\tau^{2}\left[\exp \left(\sigma^{2}\right)-1\right] \times \exp \left(\sigma^{2}\right)$, and

$$
s d(T)=\tau\left[\exp \left(\sigma^{2}\right)-1\right]^{1 / 2} \exp \left(\sigma^{2} / 2\right) .
$$

Hazard function. The hazard function (or "failure rate") of the power-lognormal distribution is

$$
h(t ; \mu, \sigma, \rho) \equiv g(t ; \mu, \sigma, \rho) / \bar{G}(t ; \mu, \sigma, \rho)=\rho \cdot h(t ; \mu, \sigma, 1) ;
$$

here $h(t ; \mu, \sigma, 1) \equiv(1 / \sigma t) \phi\{[\ln (t / \tau)] / \sigma\} / \Phi\{-[\ln (t / \tau)] / \sigma\}$ is the lognormal hazard function and $\tau=\exp (\mu)$ is the scale parameter. Rewritten

$$
h(t ; \mu, \sigma, \rho)=\rho \cdot h(r ; 0, \sigma, 1)
$$

where $r=t / \tau$. Figure 4.4 shows $h(r ; 0, \sigma, 1)$ for selected values of $\sigma$. These are a multiple of the lognormal hazard functions. Thus, they have the same behavior as lognormal hazard functions. In particular, they are all zero at $t=0$, increase to a maximum, and then decrease back toward zero with increasing time. For most $\sigma$ values seen in practice, the maximum occurs in the far upper tail. Thus, for most practical purposes, the failure rate increases as the population ages.

จno=1

s10re= $0.2 \quad 0.4 .0 .7 .1 .0$

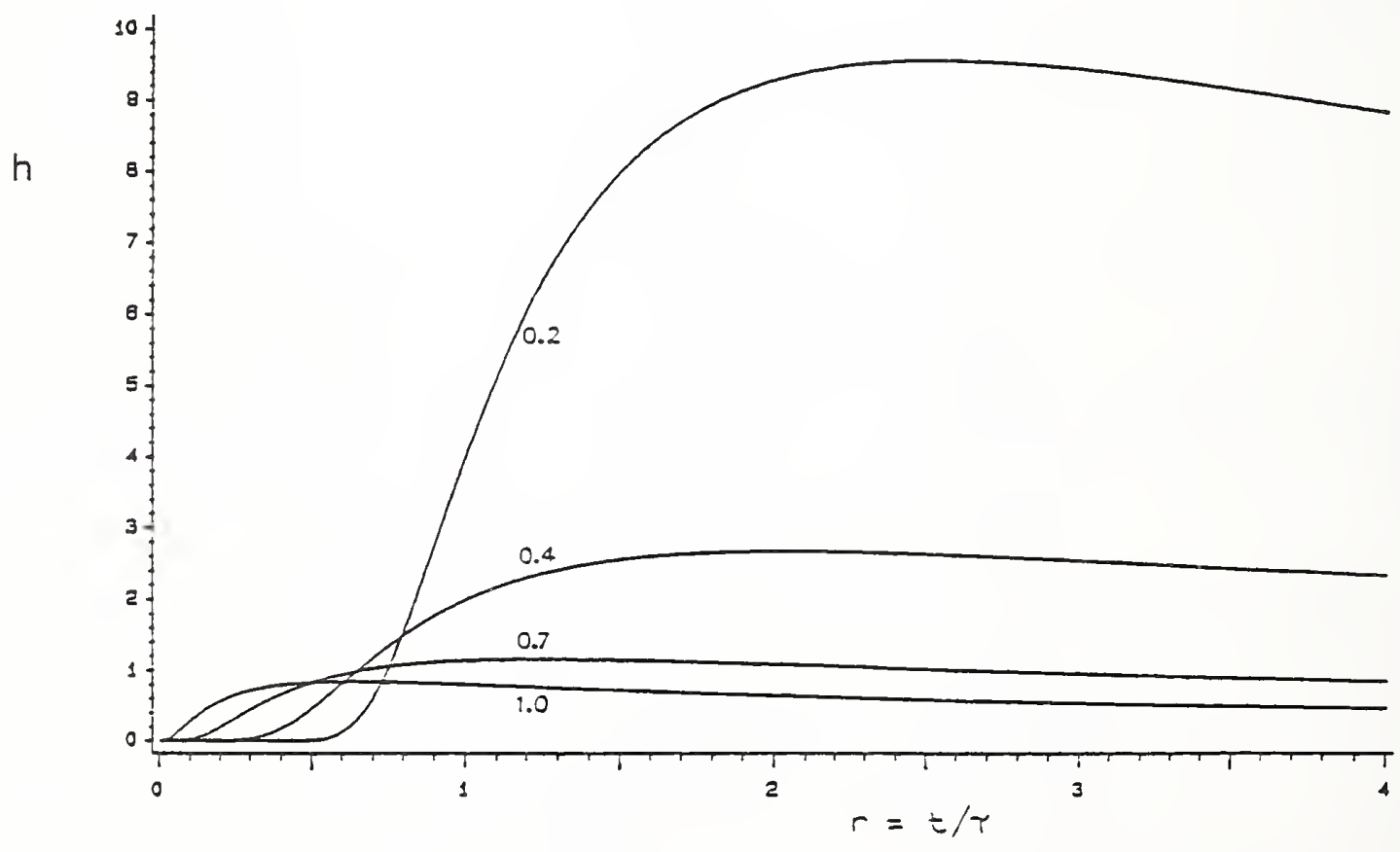

Fig. 4.4 Power-lognormal hazard functions. 


\section{MAXIMUM LIKELIHOOD THEORY FOR FITTING THE POWER-(LOG)NORMAL MODEL TO DATA}

\subsection{Introduction}

Purpose. This section presents maximum likelihood (ML) theory for fitting the power(log)normal model to data. As explained in detail by Nelson $(1982,1990)$, the ML method has good properties including

- It is versatile; that is, it applies to most models and types of data including censored and interval data.

- It has good statistical properties; namely, the method yields good estimators and confidence limits.

Overview. The contents of this section are:

- Specimen and sample likelihoods (Section 5.2),

- Maximum likelihood estimates (Section 5.3),

- Fisher and covariance matrices (Section 5.4), and

- Confidence limits (Section 5.5).

\section{$5.2 \quad$ Likelihoods}

Introduction. This section presents specimen and sample (log) likelihoods for the power(log)normal model and observed, left censored, right censored, and interval data. Terminology for life data is used, but the model may be fitted to strength and other types of data. Needed background is in Section 3 on the power-normal distribution. As background, the following paragraph describes types of sample data.

Data. The types of censored life data are defined here. For specimen $i$ with size $S_{i}$, failure may occur at an exactly observed failure age (life) $y_{i}$. If an age $t_{i}$ is from a power-lognormal distribution, then one uses the log age $y_{i}=\ln \left(t_{i}\right)$ throughout. If the specimen has run a time $y_{i}$ without failure, then the failure age is beyond and is censored on the right at age $y_{i}$; then $y_{i}$ is called the survival age without failure. If the failure occurs at some unknown age before a known first inspection age $y_{i}$, then the failure age is censored on the left at age $y_{i}$. If the failure occurs at some unknown age between two adjoining inspection ages $\left(y_{i}<y_{i}\right)$, then the failure age is interval censored and in the interval $\left(y_{i}, y_{i}\right)$. For a specimen, each such type of data has a corresponding likelihood for the power-(log)normal model, as described in the following paragraphs.

Observed. The likelihood $L_{i}$ for specimen $i$ of size $S_{i}$ that has an observed failure at (log) age $y_{i}$ is the probability density (3.7); namely,

$$
L_{i}=f\left(y_{i}, S_{i} ; \mu, \sigma, \kappa\right)=\left(\kappa S_{i} / \sigma\right) \phi\left[\left(y_{i}-\mu\right) / \sigma\right]\left\{\Phi\left[-\left(y_{i}-\mu\right) / \sigma\right]\right\}^{\kappa S_{i}-1} .
$$

This and other likelihoods are functions of the data $y_{i}$ and $S_{i}$ and the model parameters $\mu, \sigma$, and $k \equiv 1 / S_{O}$ where $S_{O}$ is the size of specimens with a (log) normal distribution. The log likelihood is

$$
\mathscr{L}_{i} \equiv \ln \left(L_{i}\right)=\ln \left(\kappa S_{i} / \sigma\right)-\ln (2 \pi)^{1 / 2}-\frac{1}{2}\left[\left(y_{i}-\mu\right) / \sigma\right]^{2}-\left(\kappa S_{i}-1\right) H\left[\left(y_{i}-\mu\right) / \sigma\right]
$$


here

$$
H(z) \equiv-\ln [\Phi(-z)]
$$

is the cumulative hazard function of the standard normal distribution.

Right censored. The likelihood $L_{i}$ for specimen $i$ of size $S_{i}$ that is right censored at $(\log )$ age $y_{i}$, its current unfailed age, is the survival probability (3.1); namely,

$$
L_{i}=\left\{\Phi\left[-\left(y_{i}-\mu\right) / \sigma\right]\right\}^{\kappa S_{i}} .
$$

The log likelihood is

$$
\mathscr{L}_{i}=-\kappa S_{i} H\left[\left(y_{i}-\mu\right) / \sigma\right]
$$

Left censored. The likelihood for specimen $i$ of size $S_{i}$ which is left censored at (log) age $y_{i}$, its inspection age after its unknown failure age, is the failure probability (3.2); namely,

$$
L_{i}=1-\left\{\Phi\left[-\left(y_{i}-\mu\right) / \sigma\right]\right\}^{\kappa S_{i}} .
$$

The log likelihood is

$$
\mathscr{L}_{i}=\ln \left[1-\left\{\Phi\left[-\left(y_{i}-\mu\right) / \sigma\right\}^{\kappa S_{i}}\right] .\right.
$$

Interval censored. The likelihood $L_{i}$ for specimen $i$ of size $S_{i}$ which is known to have failed in the interval between inspections at ages $y_{i}<y_{i}^{\prime}$ is the probability of failure in that interval; namely,

$$
L_{i}=\left\{\Phi\left[-\left(y_{i}-\mu\right) / \sigma\right]\right\}^{\kappa S_{i}}-\left\{\Phi\left[-\left(y_{i}{ }^{\prime}-\mu\right) / \sigma\right]\right\}^{\kappa S_{i}} .
$$

The log likelihood is $\mathscr{L}_{i}=\ln \left(L_{i}\right)$.

Sample likelihood. The specimen likelihoods for a sample of $n$ specimens yield the sample likelihood below. The sample log likelihood yields ML estimates and confidence limits for model parameters and other quantities, as described in the following sections. The specimen lifetimes are assumed to be a random sample of statistically independent lifetimes from a power-(log)normal distribution. The sample likelihood is the probability of the $n$ specimen outcomes (observed, censored, or interval data). For independent specimen lifetimes, the sample likelihood $L$ is the product of the specimen likelihoods; namely,

$$
L=L_{1} \times L_{2} \times \cdots \times L_{n} .
$$

Here the sample likelihood is a function of the data values $y_{i}$ (and $y_{i}$ ) and $S_{i}$, the type of data, and the model parameters $\mu, \sigma$, and $\kappa=1 / S_{0}$. The sample log likelihood $\mathscr{L} \equiv \ln (L)$ is the sum of the specimen log likelihoods; namely,

$$
\mathscr{L}=\mathscr{L}_{1}+\mathscr{L}_{2}+\cdots+\mathscr{L}_{n} .
$$




\section{3}

\section{Maximum Likelihood Estimates}

Introduction. This section defines maximum likelihood (ML) estimates and presents related theory.

Definition. The maximum likelihood estimates for the model parameters $\mu, \sigma$, and $\kappa$ are the values $\hat{\mu}, \hat{\sigma}$, and $\hat{\kappa}$ that maximize the sample likelihood (5.9) (or equivalently that maximize (5.10)) over the range of mathematically allowed values of those parameters. For many models and types of data, the maximum likelihood estimates are unique. Also, the ML estimates $(\hat{\mu}, \hat{\sigma}, \hat{\kappa})$ have an asymptotic joint normal distribution with mean vector $(\mu, \sigma, \kappa)$, the true population values, and covariance matrix $(5.20)$ below. Nelson $(1982,1990)$ references authors who state regularity conditions that the model and data must satisfy to assure this. For some models and types of data, there may be local maxima; then the parameter values at the global maximum are the ML estimates. In many computer programs, the ML estimates are iteratively calculated by direct numerical search for the maximum of $(5.10)$; Section 6 describes such calculations. An alternative calculation involves the likelihood equations which follow.

Likelihood equations. For some models and types of data, the ML estimates can be found by the calculus method. That is, the parameter values for which the partial derivatives of the sample log likelihood with respect to each parameter all equal zero are the ML estimates. For the power-(log)normal model and complete data (all specimens have exactly observed failure ages $y_{i}$ ), the partial derivatives set equal to zero are:

$$
\begin{aligned}
& 0=\partial \mathscr{L} / \partial \mu=(1 / \sigma)\left\{\sum z_{i}+\sum\left(\kappa S_{i}-1\right) h\left(z_{i}\right)\right\}, \\
& 0=\partial \mathscr{L} / \partial \sigma=(1 / \sigma)\left\{-n+\sum z_{i}^{2}+\sum\left(\kappa S_{i}-1\right) z_{i} h\left(z_{i}\right)\right\}, \\
& 0=\partial \mathscr{L} / \partial \kappa=(n / \kappa)-\sum S_{i} H\left(z_{i}\right) .
\end{aligned}
$$

Here the standardized deviate $z_{i} \equiv\left(y_{i}-\mu\right) / \sigma$ is a function of $\mu$ and $\sigma$, and each sum runs over all $n$ specimens. These likelihood equations cannot be solved explicity for $\hat{\mu}, \hat{\sigma}$, and $\hat{\kappa}$. They must be solved iteratively.

Expectations. Mathematical expectations of various quantities appear in the ML theory below. Such expectations are defined here for complete data; Nelson $(1982,1990)$ defines them for other forms of data. Let $u\left(y_{i}\right)$ denote a given function of the random failure age $y_{i}$ of specimen $i$. Then the expectation of the random quantity $u\left(y_{i}\right)$ is

$$
E\left\{u\left(y_{i}\right)\right\} \equiv \int_{-\infty}^{\infty} u\left(y_{i}\right) f\left(y_{i} ; \mu, \sigma, k\right) d y_{i}
$$

here $f($ ) is the power-normal probability density (3.8), and the parameters $\mu, \sigma$, and $\kappa$ have the true population values.

Relationships. A useful general property of the first partial derivatives of the specimen and sample log likelihoods is that their expectations are zero. That is,

$$
\begin{aligned}
& 0=E\{\partial \mathscr{L} / \partial \mu\}=(1 / \sigma)\left[\sum E\left\{z_{i}\right\}+\sum\left(\kappa S_{i}-1\right) E\left\{h\left(z_{i}\right)\right\}\right] \\
& 0=E\{\partial \mathscr{L} / \partial \sigma\}=(1 / \sigma)\left[-n+\sum E\left\{z_{i}^{2}\right\}+\sum\left(\kappa S_{i}-1\right) E\left\{z_{i} h\left(z_{i}\right)\right\}\right] \\
& 0=E\{\partial \mathscr{L} / \partial \kappa\}=(n / \kappa)-\sum S_{i} E\left\{H\left(z_{i}\right)\right\}
\end{aligned}
$$


In these formulas, the $y_{i}$ are regarded as random quantities and the parameters are equal to their true (unknown) population values. These formulas aid in the evaluation of various expectations later. For example, for a single specimen $i$, the third (5.13) equation yields

$$
1 /\left(\kappa S_{i}\right)=E\left\{H\left(z_{i}\right)\right\} \equiv \int_{-\infty}^{\infty} H\left(z_{i}\right) f\left(y_{i} ; \mu, \sigma, \kappa S_{i}\right) d y_{i}=(1 / \sigma) \int_{-\infty}^{\infty} H\left(z_{i}\right) f\left(z_{i} ; 0,1, \kappa S_{i}\right) d z_{i} ;
$$

Here $f\left(z_{i} ; 0,1, k S_{i}\right)$ is the standard power-normal distribution with $\rho_{i}=k S_{i}$. Similarly, the first (5.13) equation yields

$$
E\left\{h\left(z_{i}\right)\right\}=E\left\{z_{i}\right\} /\left(\kappa S_{i}\right)=\left(\kappa S_{i}-1\right)^{-1}(1 / \sigma) \int_{-\infty}^{\infty} z_{i} f\left(z_{i}, 0,1, \kappa S_{i}\right) d z_{i} .
$$

The last integral is the mean of the standard power-normal distribution with $\rho_{i}=k S_{i}$. These results are used to evaluate integrals below.

\subsection{Fisher and Covariance Matrices}

Introduction. This section presents the theoretical Fisher information matrix and the theoretical asymptotic covariance matrix of the ML estimates. The subsequent estimates of these matrices are used to obtain approximate confidence limits in Section 5.5.

Second derivatives. ML theory employs the second partial derivatives of the sample log likelihood. For the power-normal model and complete data, they are:

$$
\begin{aligned}
\partial^{2} \mathscr{L} / \partial \mu^{2} & =\left(-1 / \sigma^{2}\right)\left\{n+\sum\left(\kappa S_{i}-1\right) h\left(z_{i}\right)\left[h\left(z_{i}\right)-z_{i}\right]\right\}, \\
\partial^{2} \mathscr{L} / \partial \sigma \partial \mu & =\left(-1 / \sigma^{2}\right)\left\{2 \sum z_{i}+\sum\left(\kappa S_{i}-1\right) h\left(z_{i}\right)\left[1+z_{i}\left[h\left(z_{i}\right)-z_{i}\right]\right]\right\}, \\
\partial^{2} \mathscr{L} / \partial \kappa \partial \mu & =\left(S_{i} / \sigma\right) h\left(z_{i}\right), \\
\partial^{2} \mathscr{L} / \partial \sigma^{2} & =\left(-1 / \sigma^{2}\right)\left\{-n+3 \sum z_{i}^{2}+\sum\left(\kappa S_{i}-1\right) z_{i} h\left(z_{i}\right)\left[2+z_{i}\left[h\left(z_{i}\right)-z_{i}\right]\right]\right\}, \\
\partial^{2} \mathscr{L} / \partial \kappa \partial \sigma & =(1 / \sigma) \sum S_{i} z_{i} h\left(z_{i}\right), \\
\partial^{2} \mathscr{L} / \partial \kappa^{2} & =-1 / \kappa^{2} .
\end{aligned}
$$

In these equations, the derivative of the standard normal hazard function satisfies $h^{\prime}\left(z_{i}\right)=h\left(z_{i}\right)\left[h\left(z_{i}\right)-z_{i}\right]$.

Fisher matrix. The theoretical Fisher (information) matrix is the matrix of expectations of the negative second partial derivatives; namely,

$$
\mathbf{F}=\left[\begin{array}{lll}
E\left\{-\partial^{2} \mathscr{L} / \partial \mu^{2}\right\} & E\left\{-\partial^{2} \mathscr{L} / \partial \mu \partial \sigma\right\} & E\left\{-\partial^{2} \mathscr{L} / \partial \mu \partial \kappa\right\} \\
E\left\{-\partial^{2} \mathscr{L} / \partial \sigma \partial \mu\right\} & E\left\{-\partial^{2} \mathscr{L} / \partial \sigma^{2}\right\} & E\left\{-\partial^{2} \mathscr{L} / \partial \sigma \partial \kappa\right\} \\
E\left\{-\partial^{2} \mathscr{L} / \partial \kappa \partial \mu\right\} & E\left\{-\partial^{2} \mathscr{L} / \partial \kappa \partial \sigma\right\} & E\left\{-\partial^{2} \mathscr{L} / \partial \kappa^{2}\right\}
\end{array}\right]
$$

This matrix is symmetric and a function of the parameters, which equal their true (unknown) population values here. The $M L$ estimate $\hat{\mathbf{F}}$ of this matrix is obtained by replacing the parameters with their ML estimates in (5.17). Some integrals in (5.17) have not been evaluated analytically. For the ML estimate, the integrals can be evaluated by numerical quadrature when the true unknown parameter values are replaced by their ML estimates. For censored data, general formulas for such integrals appear in Nelson 
(1982, p. 378) and require a censoring time for each specimen, even those that failed. Such a planned censoring time may not be known for field data. The theoretical matrix is useful for theoretically evaluating proposed test plans where all censoring times are specified. Another estimate of $\mathbf{F}$ that does not require all censoring times and is easier to calculate is the local estimate

$$
\mathbf{F}^{*}=\left[\begin{array}{ccc}
-\partial^{2} \hat{\mathscr{L}} / \partial \mu^{2} & -\partial^{2} \hat{\mathscr{L}} / \partial \mu \partial \sigma & -\partial^{2} \hat{\mathscr{L}} / \partial \mu \partial \kappa \\
-\partial^{2} \hat{\mathscr{L}} / \partial \sigma \partial \mu & -\partial^{2} \hat{\mathscr{L}} / \partial \sigma^{2} & -\partial^{2} \hat{\mathscr{L}} / \partial \sigma \partial \kappa \\
-\partial^{2} \hat{\mathscr{L}} / \partial \kappa \partial \mu & -\partial^{2} \hat{\mathscr{L}} / \partial \kappa \partial \sigma & -\partial^{2} \hat{\mathscr{L}} / \partial \kappa^{2}
\end{array}\right] .
$$

This is the matrix of negative second partial derivatives ((5.16) for complete data) of the sample log likelihood where the caret ${ }^{\wedge}$ indicates that the parameters are set equal to their ML estimates. For example,

$$
-\partial^{2} \hat{\mathscr{Q}} / \partial \kappa \partial \mu=-(1 / \hat{\sigma}) \Sigma S_{i} h\left(\hat{z}_{i}\right)
$$

where $\hat{z}_{i}=\left(y_{i}-\hat{\mu}\right) / \hat{\sigma}$. Nelson (1982) gives other (equivalent) formulas for the theoretical Fisher information matrix and other estimates for it. This local estimate of the Fisher matrix does not require a censoring time for each specimen, only for the censored specimens. Thus it can always be used, even when the ML estimate $\hat{\mathbf{F}}$ cannot due to unknown censoring times for failed specimens.

Covariance matrix. The theoretical asymptotic covariance matrix $\mathbf{L}$ of the ML estimates $\hat{\mu}, \hat{\sigma}$, and $\hat{\kappa}$ is the inverse of the theoretical Fisher information matrix; namely,

$$
Z \equiv\left[\begin{array}{lll}
\operatorname{Var}(\hat{\mu}) & \operatorname{Cov}(\hat{\mu}, \hat{\sigma}) & \operatorname{Cov}(\hat{\mu}, \hat{\kappa}) \\
\operatorname{Cov}(\hat{\sigma}, \hat{\mu}) & \operatorname{Var}(\hat{\sigma}) & \operatorname{Cov}(\hat{\sigma}, \hat{\kappa}) \\
\operatorname{Cov}(\hat{\kappa}, \hat{\mu}) & \operatorname{Cov}(\hat{\kappa}, \hat{\sigma}) & \operatorname{Var}(\hat{\kappa})
\end{array}\right]=\mathbf{F}^{-1}
$$

Nelson (1982, pp. 384-386) gives a heuristic argument for this result. $\mathbf{Z}$ is a function of the parameters $\mu, \sigma$, and $\kappa$ set equal to their true (unknown) population values. The ML estimate of the covariance matrix is the inverse of the ML estimate (5.17) of the Fisher matrix; namely, $\hat{\mathbf{L}}=\hat{\mathbf{F}}^{-1}$. Similarly, the local estimate of the covariance matrix is the inverse of the local estimate (5.18) of the Fisher matrix; namely, $\mathbf{Z}^{*}=\left(\mathrm{F}^{*}\right)^{-1}$. An estimate of the covariance matrix yields approximate confidence limits for parameters and functions of parameters as described in Section 5.5.

\subsection{Confidence Limits}

Introduction. This section presents approximate confidence limits for model parameters and functions of them.

Function estimate. Often one wishes to estimate some function $\theta=\theta(\mu, \sigma, \kappa)$ of the model parameters. For example, the $F$ fractile of the power-normal model for specimens of size $S$ is the function $\eta_{F}=\mu+z_{F} \sigma$ where $F^{\prime}=1-(1-F)^{1 / \kappa S}$ and $z_{F^{\prime}}=\Phi^{-1}\left(F^{\prime}\right)$. Also, for example, the "normal size" is the function $S_{0}=1 / \kappa$ of just $\kappa$. The ML estimate of the true function value $\theta=\theta(\mu, \sigma, \kappa)$ is the function evaluated at the ML estimates of the parameters; namely, $\hat{\theta}=\theta(\hat{\mu}, \hat{\sigma}, \hat{\kappa})$. For example, $\hat{F}^{\prime}=1-(1-F)^{1 / \hat{R} S}$ and $\hat{\eta}_{F}=\hat{\mu}+z_{F}, \hat{\sigma}$. Under certain regularity conditions that hold for many models and data, the asymptotic cumulative distribution of $\hat{\theta}$ for "large" samples is close to a normal cumulative 
distribution with mean $\theta$ (the true population value) and variance $\operatorname{Var}(\hat{\theta})$ given below in (5.22). This asymptotic normal distribution yields the approximate confidence limits in the next paragraph.

Confidence limits. Based on the asymptotic normal distribution of $\hat{\theta}$, lower and upper two-sided approximate $100 C \%$ normal confidence limits for the true value $\theta$ are

$$
\underline{\theta}=\hat{\theta}-z_{C} \cdot[\operatorname{Var}(\hat{\theta})]^{1 / 2}, \quad \tilde{\theta}=\hat{\theta}+z_{C} \cdot[\operatorname{Var}(\hat{\theta})]^{1 / 2}
$$

here $z_{C}$, is the standard normal $C^{\prime}=(1+C) / 2$ fractile, and $\operatorname{Var}(\hat{\theta})$ appears below in (5.22). In practice, one uses in (5.21) an estimate of $\operatorname{Var}(\hat{\theta})$ given below.

Variance. This paragraph presents the formula for the theoretical asymptotic variance $\operatorname{Var}(\hat{\theta})$. It is assumed that the function $\theta(\mu, \sigma, \kappa)$ has continuous partial derivatives with respect to the parameters $\mu, \sigma$, and $\kappa$. Then

$$
\begin{aligned}
\operatorname{Var}(\hat{\theta})= & {\left[\begin{array}{lll}
\partial \theta / \partial \mu & \partial \theta / \partial \sigma & \partial \theta / \partial \kappa
\end{array}\right] \mathbb{Z}\left[\begin{array}{l}
\partial \theta / \partial \mu \\
\partial \theta / \partial \sigma \\
\partial \theta / \partial \kappa
\end{array}\right] } \\
= & (\partial \theta / \partial \mu)^{2} \operatorname{Var}(\hat{\mu})+2(\partial \theta / \partial \mu)(\partial \theta / \partial \sigma) \operatorname{Cov}(\hat{\mu}, \hat{\sigma}) \\
& +(\partial \theta / \partial \sigma)^{2} \operatorname{Var}(\hat{\sigma})+2(\partial \theta / \partial \sigma)(\partial \theta / \partial \kappa) \operatorname{Cov}(\hat{\sigma}, \hat{\kappa}) \\
& +(\partial \theta / \partial \kappa)^{2} \operatorname{Var}(\hat{\kappa})+2(\partial \theta / \partial \kappa)(\partial \theta / \partial \mu) \operatorname{Cov}(\hat{\mu}, \hat{\kappa}) .
\end{aligned}
$$

Here the partial derivatives and variances and covariances are all evaluated at the true values of the parameters. The local (or maximum likelihood) estimate of this variance is given by (5.22) where the partial derivatives are evaluated at the ML estimates of the parameters and the variances and covariances are replaced by their local (or maximum likelihood) estimates. In practice, the local estimate is usually easier to calculate. Either estimate is used to obtain the confidence limits (5.21).

Covariance. Suppose that one wishes to estimate another function $\psi=\psi(\mu, \sigma, \kappa)$ of the model parameters. Its ML estimate $\hat{\psi}=\psi(\hat{\mu}, \hat{\sigma}, \hat{\kappa})$ and $\hat{\theta}=\theta(\hat{\mu}, \hat{\sigma}, \hat{\kappa})$ have an asymptotic joint normal cumulative distribution for large samples. That joint distribution has a mean vector $(\theta, \psi)$, the true population values, variances given by (5.22), and covariance

$$
\begin{aligned}
\operatorname{Cov}(\hat{\theta}, \hat{\psi})= & (\partial \theta / \partial \mu)(\partial \psi / \partial \mu) \operatorname{Var}(\hat{\mu})+(\partial \theta / \partial \sigma)(\partial \psi / \partial \sigma) \operatorname{Var}(\hat{\sigma})+(\partial \theta / \partial \kappa)(\partial \psi / \partial \kappa) \operatorname{Var}(\hat{\kappa}) \\
& +[(\partial \theta / \partial \mu)(\partial \psi / \partial \sigma)+(\partial \theta / \partial \sigma)(\partial \psi / \partial \mu)] \operatorname{Cov}(\hat{\mu}, \hat{\sigma}) \\
& +[(\partial \theta / \partial \sigma)(\partial \psi / \partial \kappa)+(\partial \theta / \partial \kappa)(\partial \psi / \partial \sigma)] \operatorname{Cov}(\hat{\sigma}, \hat{\kappa}) \\
& +[(\partial \theta / \partial \mu)(\partial \psi / \partial \kappa)+(\partial \theta / \partial \kappa)(\partial \psi / \partial \mu)] \operatorname{Cov}(\hat{\mu}, \hat{\kappa}) .
\end{aligned}
$$

This result is used in Section 6 for transformed parameters used for convenience in place of the natural model parameters $\mu, \sigma$, and $\kappa$.

LR limits. The approximate normal limits (5.21) are simple to calculate for most models and types of data, and they are widely used. In practice, such limits usually enclose the corresponding true value with a probability below the stated confidence $100 \mathrm{C} \%$. Calculation of likelihood ratio (LR) intervals is described by Nelson (1990, pp. 297-301). Such intervals usually have a confidence level 
closer to the stated $100 \mathrm{C} \%$. However, their computation is more complex, and they are not yet available in POWNOR and in most computer programs for ML fitting.

Improved normal limits. The approximate normal confidence limits (5.21) can often be improved by working with a transformed value $\zeta=\zeta(\theta)$ and treating its ML estimate $\hat{\zeta}=\zeta(\hat{\theta})$ as normally distributed. Then one calculates the limits

$$
\underline{\zeta}=\hat{\zeta}-z_{C} \cdot[\operatorname{Var}(\hat{\zeta})]^{1 / 2}, \quad \bar{\zeta}=\hat{\zeta}+z_{C} \cdot[\operatorname{Var}(\hat{\zeta})]^{1 / 2}
$$

Here $\operatorname{Var}(\hat{\zeta})=(\partial \zeta / \partial \theta)^{2} \operatorname{Var}(\hat{\theta})$ and is estimated as described above. Then the limits for $\theta$ are obtained by inverting the monotone function $\zeta$ ) to get $\theta=\zeta^{-1}(\zeta)$ and $\bar{\theta}=\zeta^{-1}(\zeta)$, if $\zeta(\theta)$ is an increasing function of $\theta$. Otherwise, $\theta=\zeta^{-1}(\zeta)$ and $\bar{\theta}=\zeta^{-1}(\zeta)$, if $\zeta(\theta)$ is a decreasing function of $\theta$. For example, in the power-(log)normal model, a useful transformation is $\zeta=\ln (\sigma)$. This transformation assures that the confidence limits for $\sigma$ are positive, whereas, (5.21) may yield a negative lower limit, which is unacceptable since $\sigma>0$. Such transformed limits may have a confidence level that is closer to the stated $100 \mathrm{C} \%$.

\section{NUMERICAL METHODS}

Introduction. This section describes numerical methods that facilitate the calculations involved in the ML theory in Section 5. Many formulas and ideas in Section 5 are computationally naive and must be altered as shown here. In particular, finding the ML estimates by iteratively maximizing the log likelihood is difficult unless the model is reparametrized. Even the best optimization routine has difficulty optimizing the log likelihood of a poorly parametrized model, and the natural model parameters are often a poor choice. As background, Ross (1990) discusses such numerical problems and solutions in detail, and Nelson (1982, pp. 386-395) provides further solutions. This section will interest only a few who are concerned with numerical methods.

Starting values. The following method uses percentile estimation to obtain starting values $\hat{\mu}_{0}$, $\hat{\sigma}_{0}$, and $\hat{\kappa}_{0}$ for the ML iteration. The method employs three selected sample order statistics $y_{i}<y_{i}$, $<$ $y_{i}$ "with corresponding probability plotting positions $P<P^{\prime}<P^{\prime \prime}$. The next paragraph deals with the choice of $i, i^{\prime}$, and $i^{\prime \prime}$. For a complete sample of $n$ specimens of size $S$, the plotting position of order statistic $i$ is $P_{i}=(i-0.5) / n$. The $P$ fractile of the power-normal distribution is

$$
\eta_{P}=\mu+z_{x} \sigma
$$

where $\pi=1-Q^{1 / \kappa S}$ and $Q=1-P$. For specified

$$
Q=1-P=(n-i+0.5) / n, \quad Q^{\prime}=1-P^{\prime}=\left(n-i^{\circ}+0.5\right) / n, Q^{-}=1-P^{-}=\left(n-i^{*}+0.5\right) / n,
$$

the differences of the corresponding fractiles are

$$
\eta_{P^{-}}-\eta_{P^{\circ}}=\left(z_{\pi^{-}}-z_{\pi^{\circ}}\right) \sigma, \eta_{P^{-}}-\eta_{P}=\left(z_{\pi^{\circ}}-z_{\pi}\right) \sigma .
$$

The ratio of these equations yields

$$
\left(\eta_{P^{-}}-\eta_{P^{\circ}}\right) /\left(\eta_{P^{-}}-\eta_{P}\right)=\left[z_{1-Q^{\prime 1 / \kappa} S}-z_{1-Q^{1 / \kappa} S}\right] /\left[z_{1-Q^{.1 / \kappa} S}-z_{\left.1-Q^{1-\kappa} S\right]}\right.
$$

Substitute the order statistics $y_{i}, y_{i^{\prime}}$, and $y_{i}$ for the corresponding fractiles $\eta_{P}, \eta_{P^{\prime}}$, and $\eta_{P^{\prime \prime}}$ on the left side of this equation, and iterate on $b=1 /(\kappa S)$ on the right side to find the value $b_{0}$ that satisfies this 
Then use $\hat{\kappa}_{0}=1 /\left(b_{0} S\right)$ as the starting value. Then substituting the order statistics $y_{i}$ and $y_{i}$, for $\eta_{P}$ and $\eta_{P}$ into (6.3) yields a starting value $\hat{\sigma}_{0}$; namely,

$$
\hat{\sigma}_{0}=\left(y_{i^{\prime}}-y_{i}\right) /\left(z_{\pi^{\prime}}-z_{\tilde{z}}\right)
$$

where $\hat{\pi}=1-Q^{b}$ and $\hat{\pi}^{\prime}=1-Q^{b^{b}}$. Finally, substituting the order statistic $y_{i}$ for $\eta_{P}$ in (6.1) yields

$$
\hat{\mu}_{0}=y_{i}-z_{\hbar} \hat{\sigma}_{0} \text {. }
$$

These starting values are the percentile estimates based on the order statistics $y_{i}, y_{i^{\prime}}$, and $y_{i^{\prime \prime}}$. These starting values can be used to calculate starting values for another parametrization of the model. This method extends readily to multiply (right) censored samples, but must be adapted to interval data. Treat each failure in an interval as if it occurred at the midpoint of its interval, and use the method above. This yields crude starting values.

Order statistics. For a complete sample, the order statistics used in the previous paragraph are chosen as follows. For a (log)normal distribution $(\rho=1)$, the asymptotically "best" (as $n \rightarrow \infty)$ two order statistics for estimating $\sigma$ have ranks $i$ and $i$ " satisfying

$$
0.0694=(i-0.5) / n, \quad 0.9306=\left(i^{-}-0.5\right) / n \text {. }
$$

The solutions are

$$
i=0.0694 n+0.5, i^{-}=0.9306 n+0.5,
$$

which each must be rounded to the nearest integer. Use the rounded values in (6.8) to calculate the plotting positions in the previous paragraph. For the middle order statistic use the sample median; that is, $i^{\prime}=n / 2$ for even $n$ and $i^{\prime}=(n+1) / 2$ for odd $n$, and $P^{\prime}=Q^{\prime}=0.50$. For a (multiply) censored sample, calculate plotting positions for the failure times as described by Nelson (1982, Chap. 4), and use one of the early failures $P_{i}$, one of the last failures $P_{i^{\prime \prime}}$ and one about midway between them, $P_{i}=\left(P_{i}\right.$ $\left.+P_{i \prime \prime}\right) / 2$.

Iteration parameters. The theory in Sections 3, 4, and 5 employs the natural model parameters $\mu, \sigma$, and $\rho$ (or equivalently $S_{0}$ or $\kappa=1 / S_{0}$ ). These parameters are mathematically convenient and easy to interpret, especially $\mu$ and $\sigma$, which are well understood as parameters of the normal distribution $(\rho=1)$. For purposes of calculating ML estimates by optimizing a sample log likelihood, other parameters work better. POWNOR uses the parameters $\mu, \delta=\ln (\sigma)$, and $\omega=\ln \left(S_{0}\right)$ in the $\log$ likelihood. That is, $\exp (\delta)$ replaces $\sigma$, and $\exp (\omega)$ replaces $S_{0}$. The new parameters all range from $-\infty$ to $+\infty$. Thus the optimization with respect to $\mu, \delta$, and $\omega$ is unconstrained; whereas iterated values of $\sigma$ and $S_{0}$ would have to be constrained to be positive, which complicates the optimization calculations. Moreover, confidence limits $(\underset{\delta}{\delta}, \tilde{\delta})$ and $(\omega, \tilde{\omega})$ convert into positive confidence limits $\sigma=\exp (\underline{\delta}), \tilde{\sigma}=\exp (\tilde{\delta})$, $\underline{S}_{0}=\exp (\underset{\omega}{\omega})$, and $\tilde{S}_{0}=\exp (\tilde{\omega})$ avoiding negative lower limits. Also, for various models, such transformations tend to give actual confidence levels that are closer to the intended levels than untransformed parameters (Nelson (1982, p.393)).

Optimization of the log likelihood. The ML estimates of the model parameters are the parameter values that maximize the sample log likelihood, which is calculated as described in Section 5.2. The program employs the Powell (1964) method to iteratively search for the values of the model parameters that maximize the log likelihood. This method does not use the explicit derivatives of the log likelihood function taken with respect to the parameters. The user must provide the Powell method with starting 
values for the ML estimates of model parameters. A method to obtain starting values for the ML iteration is given above.

Trimmed standardized deviates. In searching for the parameters that maximize the log likelihood function, POWNOR works with standardized deviates $z_{i} \equiv\left(y_{i}-\hat{\mu}\right) / \hat{\sigma}$ of observations. If the starting values of the model parameters are far from their ML estimates, the standardized deviates are large. Such large values can cause overflow problems and slow down or prevent the convergence of the iterative method. POWNOR avoids this through the use of trimmed standardized deviates, as described in Nelson (1982, p. 394). POWNOR uses the trimmed standardized deviates until the iteration is close to converging. Then the program switches to using the (untrimmed) standardized deviates of the observations and completes the ML iteration.

Standard normal cdf and its inverse. As described in Section 5, the log likelihood function for a censored value contains the standard normal cdf. POWNOR uses the double-precision IMSL (1987) subroutine DNORDF to evaluate the standard normal cdf.

Fisher matrix. After POWNOR obtains the ML estimates, it calculates the local estimate of the Fisher (information) matrix. The elements of this matrix are the negative second partial derivatives of the log likelihood taken with respect to the (transformed) model parameters and evaluated at their ML estimates. POWNOR uses the STATLIB subroutine HESS which obtains these derivatives numerically by a perturbation calculation using only the values of the log likelihood function.

Covariance matrix. The local estimate of the covariance matrix for the ML estimates of the model parameters is the inverse of the local estimate of the Fisher matrix. The program uses the IMSL (1987) subroutine DLINDS to invert the local estimate of the Fisher matrix.

Confidence limits. The program calculates approximate confidence limits for $\mu, \ln (\sigma)$, and $\ln \left(S_{0}\right)$, using their ML estimates and local estimates of their variances. This assures positive lower limits for $\sigma$ and $S_{0}$. Calculation of confidence limits for a distribution percentile requires the inverse of the standard normal cdf. The program uses the IMSL (1987) subroutine DNORIN to evaluate the inverse of the standard normal cdf.

Reparametrization. Numerical maximization of the log likelihood function is typically facilitated by orthogonality of the model parameters. Two parameters are defined to be orthogonal if the (asymptotic) covariance of their ML estimators is zero (Cox and Reid (1987)). Ross (1990) describes how to use the information matrix to obtain (approximately) orthogonal parameters. This reparametrization multiplies the original parameter vector by the upper triangular Cholesky decomposition of their information matrix. The routine POWNOR_ORTHO uses the information matrix for $\mu, \ln (\sigma)$, and $\ln \left(S_{0}\right)$ evaluated at the current values of these parameters in such reparametrization. If the current values of the parameters are already close to the ML estimates, the transformed parameters are then approximately orthogonal. The routine POWNOR_ORTHO usually maximizes the log likelihood more quickly with respect to the transformed parameters. 


\section{REFERENCES}

Bury, K.V. (1975), "Distribution of Smallest Log-Normal and Gamma Extremes," Statistische Hefte 16, 105-114. In English.

Chaplin, W.S. (1880), "The Relation between the Tensile Strengths of Long and Short Bars," Van Nostrand Engineering Magazine 23, 441-444.

Cox, D.R. and Reid, N. (1987), "Parameter Orthogonality and Approximate Conditional Inference," J. Royal Statist. Soc. B 49, 1-39.

David, H.A. (1981), Order Statistics, 2nd edition, Wiley, New York, Order Dept. (800) 526-5368.

Harter, H.L. (1977)," A Survey of the Literature on the Size Effect on Material Strength," Report No. AFFDL-TR-77-11, Air Force Flight Dynamics Lab. AFSC, Wright-Patterson AFB, OH 45433. Author at 203 N. McKinley, Champaign, IL 61821.

IMSL (1987), User's Manual Stat/Library, Version 1, IMSL, Inc., 2500 ParkWest Tower One, 2500 City West Boul., Houston, TX 77042-3020, (713) 782-6060.

LaCombe, D.J. and Parks, E.L. (1986), "The Distribution of Electromigration Failures," Proceedings of the 1986 International Reliability Physics Symposium, 1-6.

Nelson, Wayne (1982), Applied Life Data Analysis, Wiley, New York, Order Dept. (800)526-5368.

Nelson, Wayne (1990), Accelerated Testing: Statistical Models, Test Plans, and Data Analyses, Wiley, New York, Order Dept. (800)526-5368.

Powell, M.J.D. (1990), "An Efficient Method for Finding the Minimum of a Function without Derivatives," Computer Journal 7, 155-162.

Ross, G.J.S. (1990), Nonlinear Estimation, Springer-Verlag, New York, Order Dept. (800) 777-4643.

Schafft, H.A., Staton, T.C., Mandel, J., and Shott, J.D. (1987), "Reproducibility of Electromigration Measurements," IEEE Trans. on Electron Devices, Vol. ED-34, No. 3 (March 1987), 673-681.

Shimizu, K. and Crow, E.L., eds. (1988), Lognormal Distributions, Marcel Dekker, New York, Order Dept. (800) 228-1160. 


\section{BIBLIOGRAPHIC DATA SHEET}

\begin{tabular}{l} 
2. PERFORLINO OROANIZATION REPORT MUMBEA \\
\hline . MUUUCATION DATE \\
MARCH 1992
\end{tabular}

TILEAND SUDTाR

A Computer Program POWNOR for Fitting the Power-Normal and -Lognormal Models to Life or Strength Data from Specimens of Various Sizes

AUTHOA(S)

Wayne Nelson, Necip Doganaksoy PERFOAMIMG ORQAMIZATION (IF JOINT OA OTHEA THAN MIST, SEE INSTRUCTONS) U.s. DEPANTMEarT Of COMMERCE MATIOMAL INSTITUTE OF STANDARDS AND TECHNOLOOY

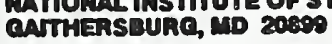

7. CONTRACT/GRANT NUMBER

Q. TYPE OF REPORT AMO PERIOD COVERED

SPONSORINO ORQAMVATION MAME ANO COMPLETE ADDAESS (STREET, CTT, STATE, ZP)

NIST

Gaithersburg, IND 20899

National Science Foundation

1800 G. Street, N.W.

Washington, D.C. 20550

American Statistical Assod. 1429 Duke Street

Alexandria, VA 22314-3402

D. SUPPLEMENTARY NOTES

11. ABSTRACT A 200-WOAD OR LESS FACTUAL SUMMAAY OF MOST SIOMIFICANT IMFOALATIOH. WF DOCUMENT IMCWDES A SIONIFICANT EIBUOGAPAY OR UTERATURE SUAVE, MEMTION IT MERE)

This report presents the power-normal and-lognormal models, which describe the effect of specimen size on the distribution of life or strength of a product or material. Such a model arises when any specimen can be regarded as a series system of smaller portions, where portions of a certain size have a normal or lognormal life (or strength) distribution. Also, this report documents the first computer program that fits this model to data (including censored and interval life data) from specimens of various sizes. The program employs maximum likelihood fitting and provides approximate confidence 1imits, as well as estimates, for model parameters, distribution percentiles, and other quantities of interest. How to run the program is explained with an analysis of data on time to electromigration fallure of aluminum conductors for microcircults.

12 KEYWORDS (6 TO 12 ENTAIES; ALPAAETICAL ORDEA; CAPTTALZE ONLY PROPER MAMES; AND SEPARATE KEY WORDS BY SEMICOLONS) censored data analysis; maximum 11kelihood fitting; power-lognormal distribution; power-normal distribution; reliability data analysis; series-system mode1

\section{AVALABIUTY}



